\title{
A Correspondence between Temporal Description Logics
}

\author{
Alessandro Artale*1 — Carsten Lutz ${ }^{* *}$ \\ * Faculty of Computer Science \\ Free University of Bozen-Bolzano (Italy) \\ artale@inf.unibz.it \\ ** Institute of Theoretical Computer Science \\ TU Dresden (Germany) \\ lutz@tcs.inf.tu-dresden.de
}

ABSTRACT. In this paper, we investigate the relationship between two decidable interval-based temporal description logics that have been proposed in the literature, $\mathcal{T} \mathcal{L}-\mathcal{A L C} \mathcal{F}$ and $\mathcal{A} \mathcal{L C F}(A)$. Although many aspects of these two logics are quite similar, the two logics suggest two rather different paradigms for representing temporal conceptual knowledge. In this paper, we exhibit a reduction from $\mathcal{T} \mathcal{L}-\mathcal{A L C} \mathcal{L}$ concepts to $\mathcal{A L C} \mathcal{F}(A)$ concepts that serves two purposes: first, it nicely illustrates the relationship between the two knowledge representation paradigms; and second, it provides a tight PSPACE upper bound for $\mathcal{T} \mathcal{L}$ - $\mathcal{A L C F}$ concept satisfiabiliy, whose complexity was previously unknown.

KEYWORDS: description logics, temporal logics, computational complexity.

\section{Introduction}

Description Logics (DLs) are a family of logic-based formalisms for representing and reasoning on conceptual knowledge, which have over the the last 20 years been successfully applied to a large number of application problems [BAA 03b]. Important characteristics of description logics are high expressivity together with sound, complete and terminating reasoning algorithms. Although expressive DLs typically have a rather high theoretical complexity (often EXPTIME-complete), highly optimized reasoners, such as FaCT [HOR 00], RACER [HAA 01], and DLP [PAT 99], have been developed and exhibit a quite impressive performance on real applications [BAA 03b].

1. The first author has been partially supported by the EU projects Sewasie, KnowledgeWeb, and Interop.

Journal of Applied Non-Classical Logics. Volume $14-\mathrm{n}^{\circ}$ 1-2/2004, pages 211 to 235 


\section{JANCL - 14/2004. Issue on Interval Temporal Logics and Duration Calculi}

Temporal extensions of logic formalisms are relevant to capture the evolving behavior of dynamic domains, and they have been extensively considered in both artificial intelligence and theoretical computer science. In particular, temporal logical formalisms have been studied and applied in areas such as specification and verification of computer programs [PNU 86, EME 90], temporal information systems [GAB 94, CHO 98, CHO 03], planning and natural language [ALL 91, ALL 94, BEN 95].

Since the incorporation of temporal aspects also plays an important role in many application areas of description logics such as reasoning about temporal database schemas [ART 99b, ART 02, ART 03] and reasoning about actions and plans [ART 98, ART 99a], in the last years an increasing interest in temporal description logic (TDL) could be observed-see [ART 01, BAA 03a] for a survey. When constructing a temporal description logic, one of the most important decisions to be made is whether time points or time intervals should be used as the underlying temporal primitive [ART 01]. As known from temporal logic and other areas of artificial intelligence, this decision has a severe impact on the expressiveness and computational properties of the resulting logic [GAB 94, GAB ar, GOR 03b, GOR 03a]. In DL research, both routes haven been taken as witnessed by a series of papers on point-based TDLs [SCH 93, WOL 98, WOL 99, STU 01, LUT 01, ART 02, GAB 03], and a number of papers on interval-based ones [SCH 90, ART 94, BET 97, ART 98, LUT ar].

Interval-based TDLs have the advantage that they provide an attractive temporal expressivitiy much richer than the expressivity of point-based TDLs. On the other hand, the computational behavior of interval-based TDLs is often problematic: even very basic formalisms often turn out to be undecidable. An important example is the interval-based TDL proposed by Schmiedel [SCH 90], which is very natural but undecidable since it contains Halpern and Shoham's (undecidable) interval-based temporal logic_called $\mathcal{H S}$ in the following — as a fragment [VEN 90, HAL 91]. Due to these computational problems, one of the prime goals of this research area has been to identify decidable TDLs that are expressive enough to allow the representation of temporal conceptual knowledge in relevant application areas.

In this paper, we are concerned with two decidable interval-based TDLs: $\mathcal{T} \mathcal{L}$ $\mathcal{A L C F}$ [ART 98], and $\mathcal{A L C \mathcal { F }}(\mathcal{D})$ [LUT 02a]. $\mathcal{T} \mathcal{L}-\mathcal{A L C \mathcal { F }}$ is close in spirit to Schmiedel's undecidable temporal DL, and thus also to the temporal logic $\mathcal{H} \mathcal{S}$. It was developed for reasoning about actions and plans [ART 98, ART 99a], and is well-suited for application domains in which objects have properties that vary over time. For example, in $\mathcal{T} \mathcal{L}-\mathcal{A} \mathcal{L C} \mathcal{F}$ we can describe the evolution of students using the following concept:

$$
\diamond(\mathrm{x}, \mathrm{y})(\mathrm{y} \text { s x).(Student@x } \sqcap \text { Bachelor-Student@y). }
$$

Here, $\mathrm{x}$ and $\mathrm{y}$ denote time intervals and (y s $\mathrm{x}$ ) states that $\mathrm{x}$ and $\mathrm{y}$ begin at the same time point, but $\mathrm{y}$ ends before $\mathrm{x}$. Thus, the described persons are students for some time interval $x$ and bachelor students for some initial sub-interval y of $x$ (since they become master's students or PhD students afterwards, which is not modeled for simplicity). 
$\mathcal{T} \mathcal{L}-\mathcal{A} \mathcal{L C} \mathcal{F}$ is equipped with a rather rich language for expressing temporal relationships that is based on the-well known Allen relations for expressing the possible relationships between time intervals [ALL 83]. In [ART 98], Artale and Franconi show that concept satisfiability and subsumption, the fundamental reasoning tasks in description logics, are both decidable for $\mathcal{T} \mathcal{L}$ - $\mathcal{A} \mathcal{L C} \mathcal{F}$. To do this, they use algorithms that first convert concepts into a certain normal form by means of a quite complex syntactic rewriting, and then apply two classical reasoning procedures, one developed for temporal constraint networks and one for description logics, to reason on the normalized concept.

The second description logic addressed in this paper, $\mathcal{A L C F}(\mathcal{D})$, is not a temporal DL in its general form. Rather, it is equipped with so-called concrete domains, which are used for representing qualities of real-world entities that are of a "concrete nature": e.g. lengths, weights, temperatures, durations, and spatial extensions [LUT 03]. The concrete domain $\mathcal{D}$ of $\mathcal{A L C F}(\mathcal{D})$ is not fixed, but rather can $\mathcal{A L C \mathcal { F }}(\mathcal{D})$ be "instantiated" with a number of different concrete domains. In [LUT 97, LUT ar], it is shown that a concrete domain $A$ based on temporal intervals and the Allen relations yields an instantiation $\mathcal{A L C F}(A)$ of $\mathcal{A L C F}(\mathcal{D})$ that is well-suited for interval-based reasoning with temporal knowledge.

The paradigm underlying the representation of temporal conceptual knowledge with $\mathcal{A L C F}(A)$ is quite different from the one of $\mathcal{T} \mathcal{L}$ - $\mathcal{A} \mathcal{L C} \mathcal{F}$. While $\mathcal{T} \mathcal{L}$ - $\mathcal{A} \mathcal{L} \mathcal{C}$ is well-suited for reasoning about objects whose properties vary over time, in $\mathcal{A L C F}(A)$ objects are associated with a fixed temporal extension that can be understood as their lifespan, and during which all of their properties remain constant. It is then possible to enforce temporal constraints on the lifespans of related objects. For example, we can define a summer semester as a semester which is properly contained in some year (in contrast to winter semesters, which overlap two years):

$$
\text { Semester } \sqcap \exists \text { in-year.Year } \sqcap \exists \text { time, (in-year o time).during. }
$$

The first conjunct states that the described objects are semesters, whereas the second conjunct states that semesters are related to the year in which they take place via the functional relation in-year. Finally, the last conjunct says that the lifespan of the semester is properly contained in the lifespan of the associated year. It has been shown that satisfiability and subsumption of $\mathcal{A L C \mathcal { F }}(A)$-concepts is decidable and PSPACEcomplete [LUT 02c].

Intuitively, the two TDLs $\mathcal{T} \mathcal{L}$ - $\mathcal{A L C \mathcal { F }}$ and $\mathcal{A L C \mathcal { C }}(A)$ are closely related: they both allow the representation of temporal conceptual knowledge based on time intervals, and they both contain the non-temporal DL $\mathcal{A L C F}$ as a proper fragment. Nevertheless, the different underlying paradigms make it surprisingly hard to relate the expressive power of the two logics. The purpose of the current paper is twofold:

1) Understand the relationship between $\mathcal{T} \mathcal{L}-\mathcal{A L C F}$ and $\mathcal{A L C F}(A)$ in terms of their expressivity;

2) Provide a tight PSPACE complexity bound for concept satisfiability in $\mathcal{T} \mathcal{L}-\mathcal{A L C F}$. 
More precisely, we start with $\mathcal{T} \mathcal{L}$ - $\mathcal{A L C} \mathcal{F}$ and show, on an intuitive level, how $\mathcal{T} \mathcal{L}$ $\mathcal{A L C \mathcal { F }}$ concepts can be translated into $\mathcal{A} \mathcal{L C} \mathcal{F}(A)$ concepts that have the same meaning. This shows how the gap between the two knowledge representation paradigms of $\mathcal{T} \mathcal{L}-\mathcal{A} \mathcal{L} \mathcal{C} \mathcal{F}$ and $\mathcal{A L C} \mathcal{F}(A)$ can be bridged. Then, we formalize the translation by polynomially reducing $\mathcal{T} \mathcal{L}-\mathcal{A L C \mathcal { F }}$ concept satisfiability to $\mathcal{A} \mathcal{L C} \mathcal{F}(A)$ concept satisfiability. Due to the known PSPACE complexity of $\mathcal{A L C \mathcal { F }}(A)$, this yields a PSPACE upper bound for $\mathcal{T} \mathcal{L}$ - $\mathcal{A} \mathcal{L C} \mathcal{F}$ concept satisfiability, which is tight. An additional advantage of the reduction is that "practicable" reasoning becomes available for $\mathcal{T} \mathcal{L}$ - $\mathcal{A} \mathcal{L} \mathcal{C} \mathcal{F}$. Indeed, all modern DL reasoners such as the ones initially mentioned are based on tableau-style reasoning procedures [BAA 00]. For the logic $\mathcal{A L C F}(A)$, such a procedure has been developed in [LUT 02c]. In contrast, no (terminating) tableau-style algorithms have yet been found for logics of the $\mathcal{T} \mathcal{L}$ - $\mathcal{A} \mathcal{L} \mathcal{F}$ family. Via our translation, the $\mathcal{A} \mathcal{L C} \mathcal{F}(A)$ decision procedure can be used for $\mathcal{T} \mathcal{L}$ - $\mathcal{A} \mathcal{L C F}$, thus replacing the less practicable reasoning methods based on syntactic rewriting.

This paper is organized as follows: in Section 2, we introduce the syntax and semantics of $\mathcal{T} \mathcal{L}-\mathcal{A} \mathcal{L C} \mathcal{F}$, together with a running example. In Section 3 , we give the syntax and semantics of $\mathcal{A L C \mathcal { F }}(A)$, and show how this logic is able to express the example introduced in Section 2. Based on this example translation, we discuss how the different paradigms of temporal-conceptual knowledge representation underlying $\mathcal{T} \mathcal{L}-\mathcal{A} \mathcal{L C} \mathcal{F}$ and $\mathcal{A} \mathcal{L C} \mathcal{F}(A)$ are related. The translation is made precise in Section 4, where we use the ideas of Section 3 to reduce $\mathcal{T} \mathcal{L}-\mathcal{A L C F}$ concept satisfiability to $\mathcal{A L C F}(A)$ concept satisfiability. In this way, we demonstrate the generality of the translation technique proposed in Section 3 and obtain a PSPACE-completeness result for $\mathcal{T} \mathcal{L}-\mathcal{A L C \mathcal { F }}$ concept satisfiability. Section 5 makes some conclusions and shows future directions.

\section{The logic $\mathcal{T} \mathcal{L}-\mathcal{A L C} \mathcal{F}$}

The temporal description logic $\mathcal{T} \mathcal{L}$ - $\mathcal{A} \mathcal{L C F}$ [ART 94, ART 98] can be viewed as a combination of the non-temporal description logic $\mathcal{A L C \mathcal { F }}$ [HOL 90] with the intervalbased temporal logic $\mathcal{H S}$ of Halpern and Shoham [HAL 91]. However, to obtain decidable reasoning problems, $\mathcal{T} \mathcal{L}$ - $\mathcal{A} \mathcal{L C} \mathcal{F}$ allows only existential temporal quantifiers, but no universal temporal quantifiers - thus including only a fragment of $\mathcal{H} \mathcal{S}$. Technically, $\mathcal{T} \mathcal{L}-\mathcal{A} \mathcal{L C} \mathcal{F}$ can be regarded as a decidable fragment of first-order interval temporal logic.

The combinatory character of $\mathcal{T} \mathcal{L}-\mathcal{A L C F}$ is reflected by its syntax, which is divided into the temporal part $\mathcal{T} \mathcal{L}$ and the atemporal part $\mathcal{A L C \mathcal { F }}$. We fix countably infinite and pairwise disjoint sets of atomic concepts, roles, features, parametric features, and temporal variables. Then, $\mathcal{T} \mathcal{L}-\mathcal{A L C \mathcal { F }}$ concepts are built following the syntax rules in Figure 1. In the figure and throughout this paper, we use

- $A$ to denote atomic concepts,

- $C, D$ to denote (temporal) $\mathcal{T} \mathcal{L}-\mathcal{A L C} \mathcal{F}$ concepts, 


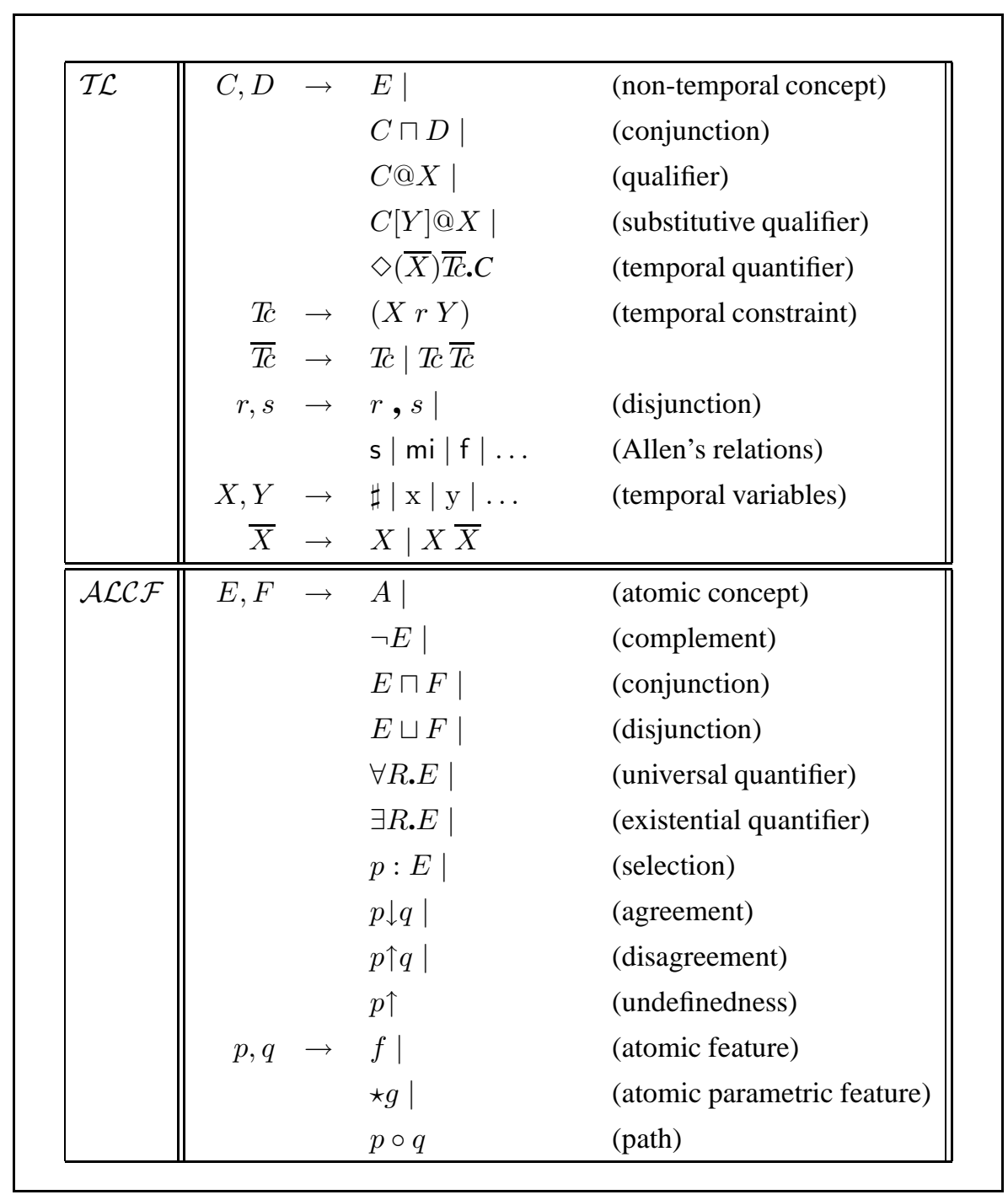

Figure 1. Syntax rules for the description logic $\mathcal{T} \mathcal{L}-\mathcal{A L C F}$ 
- E, $F$ to denote (non-temporal) $\mathcal{A L C F}$ concepts,

$-R$ to denote roles,

- $f$ to denote (non-parametric) features,

$-\star g$ to denote parametric features,

$-p$ and $q$ to denote paths, i.e. finite sequences $\gamma_{1} \circ \cdots \circ \gamma_{k}$, where each $\gamma_{i}$ is a feature or a parametric feature,

- $X, Y$ to denote temporal variables, and

$-r, s$ to denote (Allen's) interval relations.

The $\star$ symbol is not intended as an operator, but only used to distinguish parametric from non-parametric features. For the basic temporal interval relations, Allen's notation [ALL 83] is used: before (b), meets $(m)$, during $(d)$, overlaps (o), starts (s), finishes (f), equal (=), after (a), met-by (mi), contains (di), overlapped-by (oi), startedby (si), and finished-by (fi).

Due to the wealth of expressive means, a first encounter with $\mathcal{T} \mathcal{L}-\mathcal{A L C F}$ 's syntax can be slightly confusing. We will give some intuitive examples after introducing the semantics. However, an in-depth introduction to knowledge representation with $\mathcal{T} \mathcal{L}-\mathcal{A} \mathcal{L C F}$ is out of the scope of this paper, and we refer the interested reader to [ART 98]. We should also like to note that the purpose of many of $\mathcal{T} \mathcal{L}-\mathcal{A L C F}$ 's operators is to allow an intuitive representation of temporal knowledge. Technically, they can be viewed as syntactic sugar: $\mathcal{T} \mathcal{L}-\mathcal{A L C F}$ concepts can be converted into equivalent ones in a quite convenient normal form, which is introduced in Section 4.

The core of the temporal part of $\mathcal{T} \mathcal{L}-\mathcal{A} \mathcal{L C F}$ is constituted by the temporal existential quantifier " $\diamond$ " and by the "@" operator. The $\diamond$ operator introduces temporal variables that stand for time intervals, and relates such variables via temporal constraints based on the Allen relations. Then the @ operator allows to specify which concepts are "true" at intervals denoted by temporal variables. The special temporal variable $\sharp$, usually called now, is intended as the reference interval and cannot be bound by the temporal quantifier $(\diamond)$. Thus, $\sharp$ is a free temporal variable in each $\mathcal{T} \mathcal{L}-\mathcal{A} \mathcal{L C} \mathcal{F}$ concept in which it occurs. In the following, we only admit concepts that have no variables except $\sharp$ as their free variable.

$\mathcal{T} \mathcal{L}-\mathcal{A} \mathcal{L C F}$ is provided with a two-dimensional semantics, which is defined in several steps. We start with assuming a temporal structure $\mathcal{T}=(\mathcal{P},<)$, where $\mathcal{P}$ is a set of time points and $<$ is a linear, unbounded, and dense order on $\mathcal{P}$. The interval set of a structure $\mathcal{T}$ is defined as the set $\mathcal{T}_{<}^{\star}$ of all closed proper intervals $[u, v] \doteq\{x \in \mathcal{P} \mid u \leq x \leq v, u \neq v\}$ in $\mathcal{T}$. An interpretation $\mathcal{I} \doteq\left\langle\mathcal{T}_{<}^{\star}, \Delta^{\mathcal{I}},{ }^{\mathcal{I}}\right\rangle$ consists of

- a set $\mathcal{T}_{<}^{\star}$ (the interval set of the selected temporal structure $\mathcal{T}$ ),

- a set $\Delta^{\mathcal{I}}$ (the domain of $\mathcal{I}$ ), and

- a function ${ }^{\mathcal{I}}$ (the interpretation function of $\mathcal{I}$ ), which gives a meaning to atomic concepts, roles, features and parametric features: 


$$
\begin{aligned}
& A^{\mathcal{I}} \subseteq \mathcal{T}_{<}^{\star} \times \Delta^{\mathcal{I}} ; \quad R^{\mathcal{I}} \subseteq \mathcal{T}_{<}^{\star} \times \Delta^{\mathcal{I}} \times \Delta^{\mathcal{I}} ; \\
& f^{\mathcal{I}}:\left(\mathcal{T}_{<}^{\star} \times \Delta^{\mathcal{I}}\right) \stackrel{\text { partial }}{\longmapsto} \Delta^{\mathcal{I}} ; \quad \star g^{\mathcal{I}}: \Delta^{\mathcal{I}} \stackrel{\text { partial }}{\longmapsto} \Delta^{\mathcal{I}}
\end{aligned}
$$

Note the relationship between roles, features, and parametric features: first, features are simply roles that are required to be functional; second, parametric features differ from features in being independent from time, i.e., they are (temporally) global functional roles.

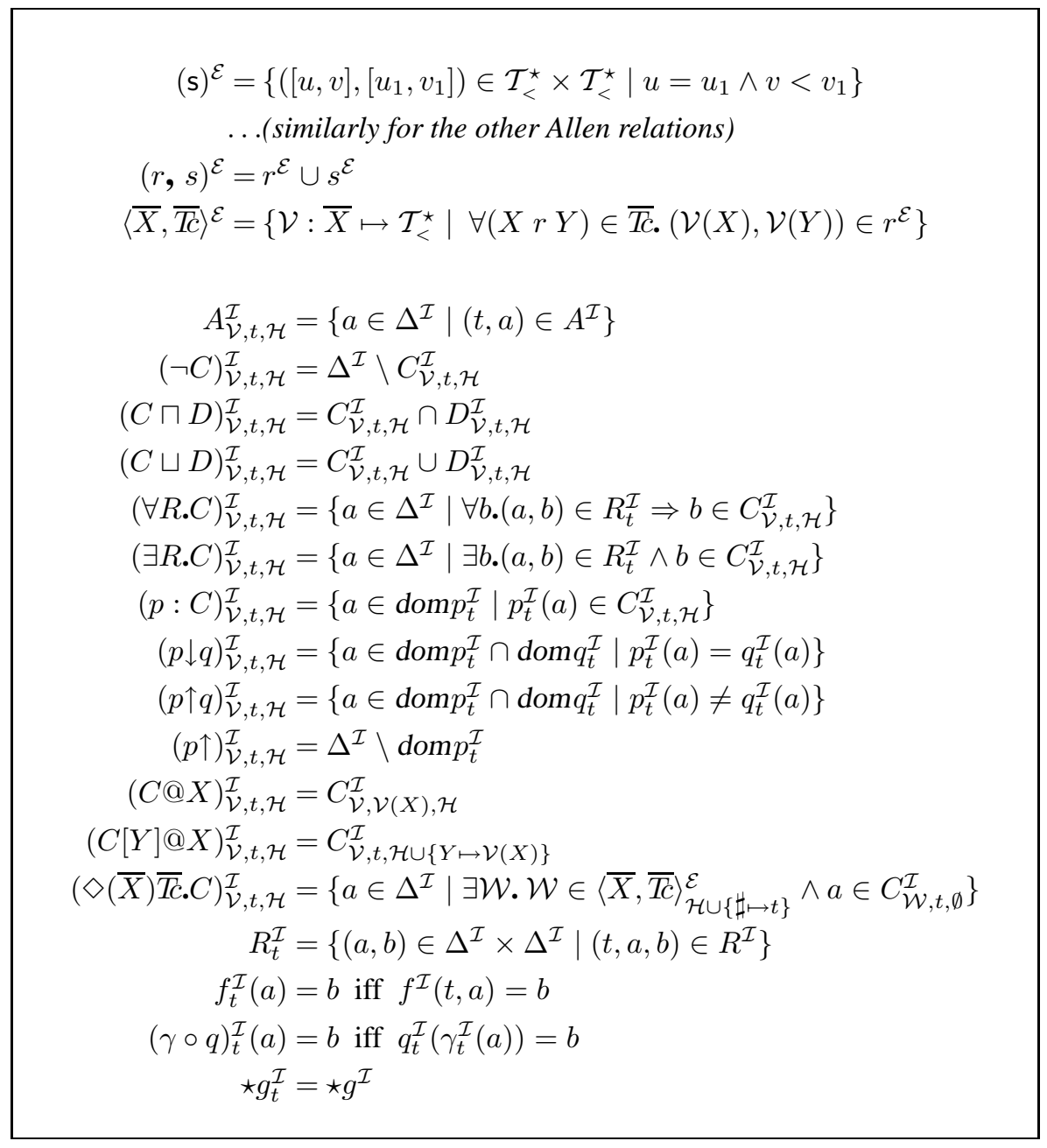

Figure 2. The $\mathcal{T} \mathcal{L}-\mathcal{A L C F}$ semantics

The second step in defining $\mathcal{T} \mathcal{L}-\mathcal{A} \mathcal{L C F}$ 's semantics consists of dealing with temporal constraint networks that occur inside the $\diamond$ operator. These networks are one of 
the most common formalisms for temporal reasoning in AI, see e.g. [ALL 83, VIL 90, NEB 95]. Formally, a temporal constraint network is a labeled directed graph $\langle\bar{X}, \overline{T c}\rangle$, where $\bar{X}$ is a set of variables representing the nodes and $\overline{T_{c}}$ is a set of temporal constraints representing the labeled edges as defined in Figure 1. The semantics of temporal constraint networks is defined using variable assignments, i.e. total functions $\mathcal{V}: \bar{X} \mapsto \mathcal{T}_{<}^{\star}$ associating an interval to each temporal variable from a set $\bar{X}$. As defined by the temporal interpretation function.$^{\mathcal{E}}$ in the upper half of Figure 2, an interpretation of a temporal constraint network is a set of variable assignments that satisfy the temporal constraints. The notation $\langle\bar{X}, \bar{T} \bar{C}\rangle_{\left\{x_{1} \mapsto t_{1}, x_{2} \mapsto t_{2}, \ldots\right\}}^{\mathcal{E}}$, used to interpret concept expressions in the next step, denotes the subset of $\langle\bar{X}, \overline{T c}\rangle^{\mathcal{E}}$ where the variable $x_{i}$ is mapped to the interval value $t_{i}$.

We can now perform the last step of defining $\mathcal{T} \mathcal{L}-\mathcal{A} \mathcal{L C F}$ 's semantics. The interpretation $C_{\mathcal{V}, t, \mathcal{H}}^{\mathcal{I}}$ of a $\mathcal{T} \mathcal{L}-\mathcal{A} \mathcal{L C} \mathcal{F}$ concept $C$ with free variables $x_{1}, \ldots, x_{k}$ (possibly including $\sharp$ ) is based on

- a variable assignment $\mathcal{V}$ such that $x_{1}, \ldots, x_{k}$ are in the domain of $\mathcal{V}$,

- an interval $t \in \mathcal{T}_{<}^{\star}$, and

- an assignment constraint $\mathcal{H}=\left\{y_{1} \mapsto t_{1}, \ldots\right\}$ with $y_{i}$ variable and $t_{i} \in \mathcal{T}_{<}^{\star}$.

The exact details of defining the interpretation of $\mathcal{T} \mathcal{L}-\mathcal{A L C F}$ concepts can be found in the lower part of Figure 2.

Intuitively, the interpretation $C_{\mathcal{V}, t, \mathcal{H}}^{\mathcal{I}}$ of a $\mathcal{T} \mathcal{L}-\mathcal{A L C F}$ concept $C$ is the set of elements of the domain which are of type $C$ at the time interval $t$, with the assignment for the free temporal variables in $C$ given by $\mathcal{V}$ (c.f. the definition of $(C @ X)_{\mathcal{V}, t, \mathcal{H}}^{\mathcal{I}}$ ) and with the assignment of variables in the scope of the outermost temporal quantifiers constrained by $\mathcal{H}$. The natural interpretation function $C_{t}^{\mathcal{I}}$, being equivalent to the interpretation function $C_{\mathcal{V}, t, \mathcal{H}}^{\mathcal{I}}$ with any $\mathcal{V}$ such that $\mathcal{V}(\sharp)=t$, and $\mathcal{H}=\emptyset$, is introduced as an abbreviation. An interpretation $\mathcal{I}$ is a model for a concept $C$ if, for some $t \in \mathcal{T}_{<}^{\star}$, $C_{t}^{\mathcal{I}} \neq \emptyset$. If a concept has a model, then it is satisfi able, otherwise it is unsatisfi able.

We will now informally discuss the intended meaning of $\mathcal{T} \mathcal{L}-\mathcal{A L C F}$ concepts. As already noted, a central role is played by the temporal existential quantifier " $\diamond$ " and the temporal qualification operator "@". For example, to represent all the objects that satisfy a concept $C$ at a time interval that is after the "current interval", we can write

$$
\diamond(x)(x \mathrm{a} \sharp) \cdot(C @ x) .
$$

Here, the $\diamond$ operator introduces the new variable $x$ and ensures that the time interval it denotes is located after the current interval $\sharp$. Then, the @ operator "evaluates" $C$ at $x$ thus ensuring that $C$ holds at the time interval denoted by $x$. 
Let us now consider some more interesting examples from the well-known blocks world domain. First, we define a concept representing the action of stacking a block on top of another block ${ }^{1}$.

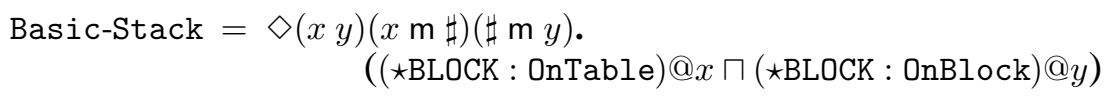

Basic-Stack denotes any action involving a $\star$ BLOCK that was once OnTable and then $\mathrm{OnBlock}$. The parametric feature $\star$ BLOCK plays the role of formal parameter of the action, mapping any individual action of type Basic-Stack to the block to be stacked, independently from time. The $\sharp$ interval can be understood as the occurring time of the stacking action. The temporal constraints $(x \mathrm{~m} \sharp)$ and $(\sharp \mathrm{m} y)$ state that the interval $x$ should meet the interval $\sharp$ - the occurrence interval of the action type Basic-Stack—and that $\sharp$ should meet $y$.

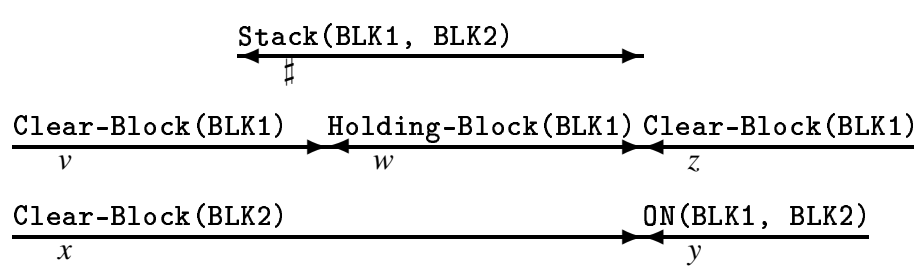

Figure 3. Temporal dependencies in the defi nition of the Stack action

To illustrate the expressive power of other $\mathcal{T} \mathcal{L}$ - $\mathcal{A} \mathcal{L C} \mathcal{F}$ constructors, let us now refine the Basic-Stack example. Figure 3 shows the temporal configuration induced by the stacking action in some more detail: a stacking action involves two blocksBLK1 and BLK2 - which should be both clear at the beginning; the central part of the action consists of grasping one block; at the end, the blocks are one on top of another, and the bottom one is no longer clear. The formal definition of the action Stack is:

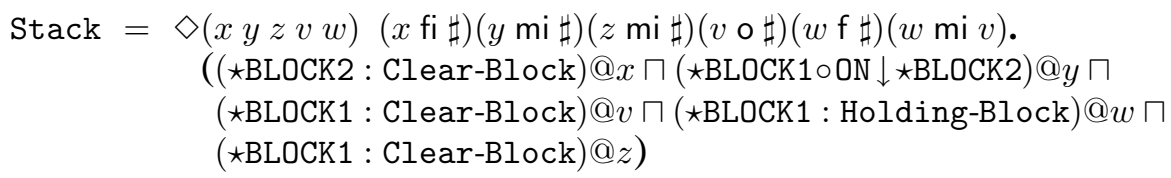

Apart from providing a more fain-grained modeling, the new definition of stacking uses the feature agreement constructor: $(\star B L O C K 1 \circ 0 \mathrm{~N} \downarrow \star \mathrm{BLOCK} 2) @ y$ indicates that, at interval $y$, the object ON which $\star B L O C K 1$ is placed is $\star B L O C K 2$. Note that the world states described at the intervals denoted by $v, w, z$ are the result of an action of grasping a previously clear block:

1. In this paper, equalities are used only for introducing names for complex concepts. Such equalities are thus not intended to denote so-called TBoxes, which are frequently used with description logics. Please refer to Section 5 for a brief discussion of reasoning under TBoxes. 


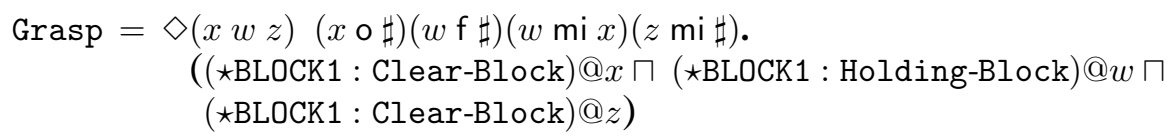

The Stack action can be redefined by making use of the Grasp action:

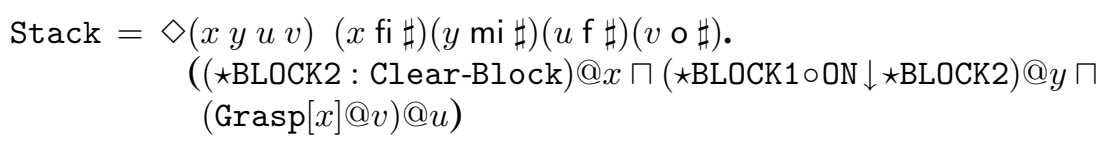

The temporal substitutive qualifier $(\operatorname{Grasp}[x] @ v)$ renames within the defined Grasp action the variable $x$ to $v$. Thus, it is a way of establishing a coreference between two temporal variables ensuring that the temporal constraints peculiar to the renamed variable $x$ are inherited by the substituting interval $v$. Furthermore, the effect of temporally qualifying the grasping action at $u$ is that the $\sharp$ variable associated to the grasping action-referring to the occurrence time of the action itself-is bound to the interval denoted by $u$. Because of this binding on the occurrence time of the grasping action, the $\sharp$ variable in the grasping action and the $\sharp$ variable in the stacking action denote different time intervals, so that the grasping action occurs at an interval finishing the occurrence time of the stacking action.

\section{The logic $\mathcal{A L C F}(A)$}

As noted in the introduction, the temporal description logic $\mathcal{A L C F}(A)$ is obtained by taking the logic $\mathcal{A L C \mathcal { F }}(\mathcal{D})$, which provides for concrete domains, and instantiating it with a concrete domain $A$ that is based on time intervals and the Allen interval relations [LUT 97, LUT 02c, LUT ar]. For the sake of brevity, we do not introduce $\mathcal{A L C F}(\mathcal{D})$ in general (see, e.g. [LUT 02c]), but rather define it's specialization $\mathcal{A L C \mathcal { F }}(A)$ right away.

The syntax of $\mathcal{A L C} \mathcal{F}(A)$ is obtained from the syntax of $\mathcal{A L C} \mathcal{F}$ as given in Figure 1 by making the following modifications:

- $\mathcal{A L C F}(A)$ does not provide parametric features.

- $\mathcal{A L C F}(A)$ is equipped with a new sort of feature, so called temporal features.

- The temporal part of $\mathcal{A L C F}(A)$ is integrated into the language by adding the temporal concept constructor:

$$
E, F \rightarrow \exists p_{1}, p_{2} . r
$$

where $r$ is one of the Allen relations, and $p_{1}, \ldots, p_{n}$ are temporal paths-sequences $\gamma_{1} \circ \cdots \circ \gamma_{k} \circ h$ with $\gamma_{1}, \ldots, \gamma_{k}$ features, and $h$ a temporal feature.

In contrast to $\mathcal{T} \mathcal{L}-\mathcal{A L C F}$, the semantics of $\mathcal{A L C \mathcal { F }}(A)$ is not a multi-dimensional one, but rather it is very close to "classical" description logics semantics. To introduce it, we again fix a linear, unbounded, and dense temporal structure $\mathcal{T}=(\mathcal{P},<)$ - this 
structure is assumed to be the same as in the $\mathcal{T} \mathcal{L}-\mathcal{A L C \mathcal { F }}$ case. Then, an $\operatorname{ALCF}(A)$ interpretation $\mathcal{I}=\left(\Delta^{\mathcal{I}},{ }^{\mathcal{I}}\right)$ consists of a set $\Delta^{\mathcal{I}}$ (the domain), and an interpretation function $\cdot{ }^{\mathcal{I}}$ that assigns a meaning to atomic concepts, roles, features, and temporal features:

$$
\begin{aligned}
& A^{\mathcal{I}} \subseteq \Delta^{\mathcal{I}} ; \quad R^{\mathcal{I}} \subseteq \Delta^{\mathcal{I}} \times \Delta^{\mathcal{I}} ; \\
& f^{\mathcal{I}}: \Delta^{\mathcal{I}} \stackrel{\text { partial }}{\longmapsto} \Delta^{\mathcal{I}} ; \quad h^{\mathcal{I}}: \Delta^{\mathcal{I}} \stackrel{\text { partial }}{\longmapsto} \mathcal{T}_{<}^{\star}
\end{aligned}
$$

If $p=q \circ h$ is a temporal path, then $p^{\mathcal{I}}$ is defined as $h^{\mathcal{I}}\left(q^{\mathcal{I}}(\cdot)\right)$, where the meaning of atemporal paths is defined as in Figure 2. Apart from the temporal concept constructor, the interpretation of complex concepts is also determined by Figure 2-just omit the three temporal indices. The semantics of the new temporal concept constructor is given as follows:

$\left(\exists p_{1}, p_{2} . r\right)^{\mathcal{I}}=\left\{a \in \Delta^{\mathcal{I}} \mid \exists t_{1}, t_{2} \in \mathcal{T}_{<}^{\star}:\left(a, t_{1}\right) \in p_{1}^{\mathcal{I}} \wedge\left(a, t_{2}\right) \in p_{2}^{\mathcal{I}} \wedge\left(t_{1}, t_{2}\right) \in r^{\mathcal{E}}\right\}$,

where $r^{\mathcal{E}}$ is defined as in Figure 2.

Before discussing the intuitions behind $\mathcal{A L C F}(A)$, let us adopt two conventions: first, we will use parametric feature names of $\mathcal{T} \mathcal{L}-\mathcal{A L C \mathcal { F }}$ as non-temporal feature names in $\mathcal{A} \mathcal{L C} \mathcal{F}(A)$. Thus, we may write e.g. $\star$ BLOCK in an $\mathcal{A L C \mathcal { F }}(A)$ concept to denote a (non-temporal) feature. Second, in the following we will only need a single temporal feature which will be denoted with time.

Comparing the semantics of $\mathcal{T} \mathcal{L}-\mathcal{A L C \mathcal { F }}$ and $\mathcal{A L C \mathcal { F }}(A)$, the main difference is that $\mathcal{T} \mathcal{L}$ - $\mathcal{A L C} \mathcal{L}$ 's semantics is two-dimensional (i.e. based on the product of the domain and the set of time intervals), while $\mathcal{A L C \mathcal { F }}(A)$ 's semantic is not. The consequences of this difference can be summarized as follows:

- in $\mathcal{T} \mathcal{L}-\mathcal{A L C F}$, a domain element may be in the extension of a concept only w.r.t. a given time interval; moreover, objects are not associated with a "life span", but rather exist at any given time interval.

- in $\mathcal{A L C F}(A)$, concept membership of domain elements is independent of time; moreover, objects are associated with a unique life span via the time feature. ${ }^{2}$

The semantic difference induces two different paradigms for the representation of temporal conceptual knowledge. If the aim is to talk about "eternal" objects whose properties vary over time, then $\mathcal{T} \mathcal{L}$ - $\mathcal{A} \mathcal{L C} \mathcal{F}$ seems like a natural choice. On the other hand, if we want to reason about temporal entities that are associated with a unique temporal extension, then using $\mathcal{A L C \mathcal { F }}(A)$ is the better approach.

2. Or with multiple time spans if we admit more than one temporal feature. This can be very useful: consider e.g. the introduction of distinct temporal features for the life time, the youth, the work time, etc. However, in the context of $\mathcal{T} \mathcal{L}-\mathcal{A} \mathcal{L C} \mathcal{F}$ we prefer to stick to a single temporal feature. 
Despite these differences, there exists a close and natural relationship between the two temporal description logics $\mathcal{T} \mathcal{L}-\mathcal{A L C \mathcal { F }}$ and $\mathcal{A L C \mathcal { F }}(A)$. To get a first idea, let us represent the basic stack action from Section 2 in the framework of $\mathcal{A L C F}(A)$ :

$$
\begin{aligned}
\text { Basic-Stack } \doteq & \text { step }_{1}:(\star \text { BLOCK }: \text { OnTable }) \sqcap \\
& \text { step }_{2}:(\star \text { BLOCK }: \text { OnBlock }) \sqcap \\
& \exists\left(\text { step }_{1} \circ \text { time }\right),\left(\text { step }_{\sharp} \circ \text { time }\right) \cdot \mathrm{m} \sqcap \\
& \exists\left(\text { step }_{\sharp} \circ \text { time }\right),\left(\text { step }_{2} \circ \text { time }\right) \cdot \mathrm{m}
\end{aligned}
$$

The concept states that any Basic-Stack is related to three objects via the features

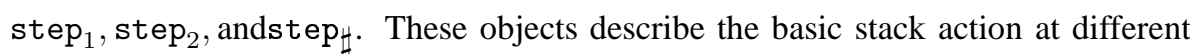
time intervals - with step $_{\sharp}$ representing the occurring time of the action. For each step, a corresponding time interval is associated by the time feature. The relation between these time intervals is described using the temporal concept constructor and resembles the temporal network in the $\mathcal{T} \mathcal{L}-\mathcal{A L C \mathcal { F }}$ definition of the basic stack. In step $_{1}$, the $\star B L O C K$ is OnTable, and in step $_{2}$ it is OnBlock. This situation is illustrated in Figure 4.

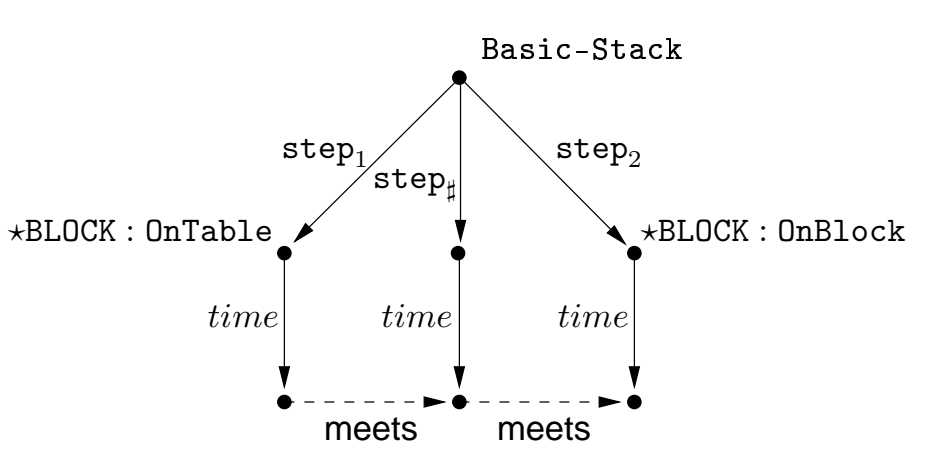

Figure 4. Model of the $\mathcal{A L C \mathcal { F }}(A)$ Basic-Stack

Comparing the two definitions of Basic-Stack, their main difference can be characterized as follows: in the $\mathcal{T} \mathcal{L}-\mathcal{A L C F}$ definition, the basic stack is represented by a single logical object, whose properties are defined separately for each temporal interval. To the contrary, in $\mathcal{A L C \mathcal { F }}(A)$, the basic stack is represented by a logical "metaobject" (the Basic-Stack object itself in the above concept definition) and a set of

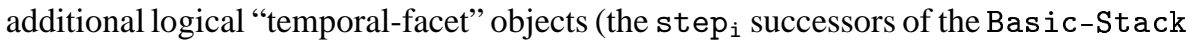
meta-object), each of which has unique properties and represents the basic stack at a unique time interval.

To reduce satisfiability of $\mathcal{T} \mathcal{L}-\mathcal{A L C} \mathcal{F}$ concepts to satisfiability of $\mathcal{A L C F}(A)$ concepts, we exploit the idea suggested by this simple example: the translation must be such that one domain element in models of the $\mathcal{T} \mathcal{L}-\mathcal{A L C \mathcal { F }}$ concepts corresponds 
to a number of domain elements in models of its $\mathcal{A L C F}(A)$ translation, i.e. one meta-object together with a number of temporal-facet objects that represent the single $\mathcal{T} \mathcal{L}-\mathcal{A} \mathcal{L} \mathcal{C} \mathcal{F}$ domain element at different time intervals. An additional difficulty is to preserve the temporal invariance of parametric features. As illustrated in the next section, this problem is solved by using the feature agreement constructor of $\mathcal{A L C F}(A)$.

\section{The reduction}

This section presents the reduction of $\mathcal{T} \mathcal{L}-\mathcal{A L C F}$ concept satisfiability to $\mathcal{A L C \mathcal { F }}(A)$ concept satisfiability. To simplify matters, we will only consider $\mathcal{T} \mathcal{L}-\mathcal{A} \mathcal{L} \mathcal{C} \mathcal{F}$ concepts in so-called existential normal form $(E N F)$. In this normal form, the only temporal operator that may occur is a single " $\diamond$ " operator, i.e. $\mathcal{T} \mathcal{L}-\mathcal{A L C F}$ concepts in ENF are of the form

$$
C=\diamond(\bar{X}) \overline{T c} \cdot Q_{0} \sqcap Q_{1} @ X_{1} \sqcap \ldots \sqcap Q_{n} @ X_{n},
$$

where $\bar{X}=\left\{X_{1}, \ldots, X_{n}\right\}$ and each $Q_{i}$ is an (atemporal) $\mathcal{A L C \mathcal { F }}$ concept. Additionally, we assume that the $\mathcal{T} \mathcal{L}$ - $\mathcal{A} \mathcal{L C} \mathcal{F}$ concepts $Q_{0}, \ldots, Q_{n}$ are in negation normal form $(N N F)$, i.e. that negation occurs only in front of concept names. In this case, we will simply say that the concept $C$ is in normal form $(N F)$. As the following proposition shows, normal form can be assumed without loss of generality.

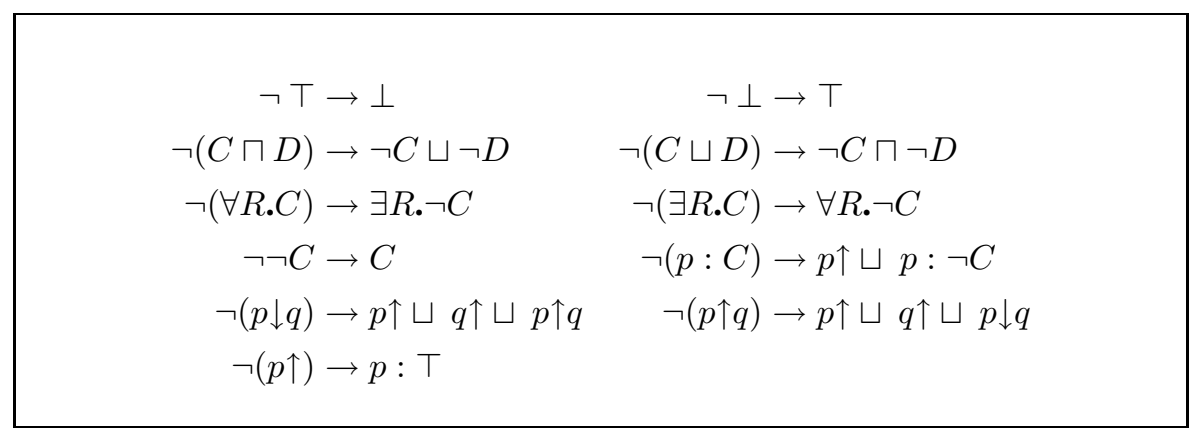

Figure 5. NNF rewrite rules

Proposition 1 (EQuivalence of NF). - Every $\mathcal{T} \mathcal{L}-\mathcal{A} \mathcal{L} \mathcal{C} \mathcal{F}$ concept $C$ can be converted in polynomial time into an equivalent concept in normal form.

PROOF. - In [ART 98], it is shown that every $\mathcal{T} \mathcal{L}-\mathcal{A} \mathcal{L C} \mathcal{F}$ concept can be converted in polynomial time to an equivalent one in ENF. We can then convert the $Q_{0}, \ldots, Q_{n}$ to NNF by exhaustively applying the rewrite rules in Figure 5. Note that this takes only polynomial time and the length of the resulting concept is polynomial in the length of the original concept. 
Let $C$ be a $\mathcal{T} \mathcal{L}-\mathcal{A L C F}$ concept in NF of the form $(*)$. To translate $C$ into an equi-satisfiable $\mathcal{A L C \mathcal { F }}(A)$ concept $\Psi(C)$, we introduce the new features $f_{0}, \ldots, f_{n}$ (corresponding to the step $_{i}$ features in Section 3 ), the new concept names $A_{i, j}$ for all $0 \leq i, j \leq n$, and the new concrete feature time. We assume w.l.o.g. that these features and concept names are not used in the $\mathcal{T} \mathcal{L}$ - $\mathcal{A} \mathcal{L C} \mathcal{F}$ concept $C$. For the remainder of this section, we use the symbol $f$ to denote features that are distinct from the reserved features $f_{0}, \ldots, f_{n}$, parametric features are denoted by $\star g$, and $\gamma$ denotes features that may or may not be parametric, but are distinct from the reserved features. To define the concept $\Psi(C)$, we need to define a number of auxiliary concepts. To start with, we need a mapping from $\mathcal{T} \mathcal{L}$ - $\mathcal{A} \mathcal{L} \mathcal{C} \mathcal{F}$ 's temporal constraint networks to $\mathcal{A L C F}(A)$ concepts.

Definition 2 (Translation of Temporal Networks). - Let $\overline{T c}$ be a temporal constraint network for the set of variables $\bar{X}=\left\{X_{0}, X_{1}, \ldots, X_{n}\right\}$, where $X_{0}=\sharp$. For each temporal constraint $(X \quad r \quad Y) \in \overline{T c}$, we defi ne an $\mathcal{A} \mathcal{L C F}(A)$ concept, $\alpha(X r Y)$, as follows:

$$
\alpha(X r Y):=\exists\left(f_{i} \circ \text { time }\right),\left(f_{j} \circ \text { time }\right) . r \quad \text { if } X=X_{i} \text { and } Y=X_{j} .
$$

Then, the translation $\alpha(\overline{T c})$ of $\overline{T c}$ is defi ned as follows:

$$
\alpha(\overline{T c}):=\prod_{(X r Y) \in \overline{T c}} \alpha(X r Y) .
$$

The remaining auxiliary concepts $-\Gamma_{C}, \Omega, \Omega^{\prime}$-are defined in Figure 6 . In the definition of $\Gamma_{C}$, we use feat $(C)$ to denote the set of all features (either non-parametric or parametric) in $C$, and $\operatorname{rol}(C)$ to denote the set of all role names in $C$.

Definition 3 (Translation OF $\mathcal{T} \mathcal{L}$ - $\mathcal{A L C \mathcal { F }}$ Concepts). - Given a $\mathcal{T} \mathcal{L}-\mathcal{A L C} \mathcal{F}$ concept $C$, its $\mathcal{A L C} \mathcal{F}(A)$ translation, $\Psi(C)$, is defi ned as:

$$
\Psi(C):=\alpha(\overline{T c}) \sqcap \Gamma_{C} \sqcap \Omega \sqcap \Omega^{\prime} .
$$

Before giving a formal proof of the fact that $\Psi(C)$ and $C$ are equi-satisfiable, let us briefly discuss the various concepts used in the reduction. The concept $\Omega$ enforces the existence of $n+1$ temporal-facet objects as $f_{i}$-successors of the root object (i.e. the object that satisfies the reduction concept $\Psi(C)$ ). Thus, this root object is a metaobject in the sense of the previous section. Furthermore, $\Omega$ ensures that, for each $i$, the temporal facet that is an $f_{i}$-successor must be a member of $\Psi_{i}\left(Q_{i}\right)$. The purpose of the $\Psi_{i}$ translation used here is to insert the $f_{i}$ features after each feature and role name used in $Q_{i}$. This is necessary since not only the root object, but also all other objects are composed of a meta-object and $n+1$ temporal facets.

The concept $\alpha(\overline{T c})$ associates a time feature to each temporal-facet of the root metaobject ensuring that the values of such time features satisfy all constraints in $\overline{T c}$. It is interesting to note that only the $f_{i}$ successors of the root meta-object are equipped with 


$$
\begin{aligned}
\Omega & :=f_{0}: \Psi_{0}\left(Q_{0}\right) \sqcap \cdots \sqcap f_{n}: \Psi_{n}\left(Q_{n}\right) \\
\Omega^{\prime}:= & \prod_{0 \leq i<j \leq n}\left(\exists\left(f_{i} \circ \text { time }\right),\left(f_{j} \circ \text { time }\right) .=\right) \rightarrow A_{i, j} \\
\Gamma_{C}:= & \prod_{0 \leq i<j \leq n} A_{i, j} \rightarrow f_{i} \downarrow f_{j} \sqcap \\
& \prod_{0 \leq i<j \leq n} A_{i, j} \rightarrow\left(\prod _ { 0 \leq k \leq n } \left(\prod_{\gamma \in \text { feat }(C)}^{\prod_{R \in \operatorname{rol}(C)}}\left(f_{k}: \gamma \uparrow \sqcup f_{k}: \gamma: A_{i, j}\right) \sqcap\right.\right. \\
& \prod_{\star g \text { used in C }}\left(\left(\cap_{i, j}^{n}\right)\right) \sqcap
\end{aligned}
$$

$$
\begin{aligned}
\Phi_{i}(\gamma) & :=\gamma \\
\Phi_{i}(p \circ \gamma) & :=\Phi_{i}(p) \circ f_{i} \circ \gamma \\
\Gamma_{C}^{i}(\gamma) & :=\gamma: \Gamma_{C} \\
\Gamma_{C}^{i}(\gamma \circ p) & :=\gamma:\left(\Gamma_{C} \sqcap f_{i}: \Gamma_{C}^{i}(p)\right) \\
\Psi_{i}(A) & :=A \\
\Psi_{i}(\neg A) & :=\neg A \\
\Psi_{i}(D \sqcap E) & :=\Psi_{i}(D) \sqcap \Psi_{i}(E) \\
\Psi_{i}(D \sqcup E) & :=\Psi_{i}(D) \sqcup \Psi_{i}(E) \\
\Psi_{i}(\exists R . D) & :=\exists R .\left(\Gamma_{C} \sqcap f_{i}: \Psi_{i}(D)\right) \\
\Psi_{i}(\forall R . D) & :=\forall R .\left(f_{i}: \Psi_{i}(D)\right) \\
\Psi_{i}(p: D) & :=\Phi_{i}(p): f_{i}: \Psi_{i}(D) \sqcap \Gamma_{C}^{i}(p) \\
\Psi_{i}(p \downarrow q) & :=\Phi_{i}(p) \downarrow \Phi_{i}(q) \sqcap \Gamma_{C}^{i}(p) \sqcap \Gamma_{C}^{i}(q) \\
\Psi_{i}(p \uparrow q) & :=\Phi_{i}(p) \uparrow \Phi_{i}(q) \sqcap \Gamma_{C}^{i}(p) \sqcap \Gamma_{C}^{i}(q) \\
\Psi_{i}(p \uparrow) & :=\Phi_{i}(p) \uparrow
\end{aligned}
$$

Figure 6. Defi nition of auxiliary concepts 
time intervals via the time feature. As we said before, all successors of such temporalfacet objects implicitly "inherit" the same temporal structure via the $\Psi_{i}$ translation.

The concept $\Gamma_{C}$ serves two purposes. First, the last row of $\Gamma_{C}$ uses feature agreements to ensure that parametric features are independent from time, i.e. if two $\operatorname{ALC\mathcal {F}}(A)$ domain elements $d_{1}$ and $d_{2}$ represent two temporal-facet of the same meta-object, then $d_{1}$ and $d_{2}$ should have the same successor for each parametric feature. Second, together with the $\Omega^{\prime}$ concept and translations $\Psi_{i}$ and $\Gamma_{C}^{i}, \Gamma_{C}$ ensures that if, for a given meta-object, two variables $X_{i}$ and $X_{j}$ denote the same time interval, then for each successor of such meta-object both $f_{i}$ and $f_{j}$ features coincide. The latter is necessary since, in $\mathcal{T} \mathcal{L}$ - $\mathcal{A} \mathcal{L} \mathcal{C} \mathcal{F}$ models, a domain element together with a time interval uniquely identifies concept membership, role membership, etc.

\section{Proof of correctness}

Throughout the proofs, we will write $\operatorname{sub}(C)$ to denote the set of all subconcepts of the concept $C$, including $C$ itself. We now establish the correctness of our reduction. For the sake of clarity, it is split into two propositions.

Proposition 4. - Let $C$ be a $\mathcal{T} \mathcal{L}$ - $\mathcal{A} \mathcal{L} \mathcal{C} \mathcal{F}$ concept in normal form. Then $\mathcal{A L C \mathcal { F }}(A)$ satisfi ability of $\Psi(C)$ implies $\mathcal{T} \mathcal{L}$ - $\mathcal{A L C \mathcal { F }}$ satisfi ability of $C$.

ProOF. - Let $\mathcal{I}$ be a model of $\Psi(C)$, and let $d_{C} \in \Psi(C)^{\mathcal{I}}$. We define $\Delta^{*}$ to be the smallest subset of $\Delta$ satisfying the following properties:

1) $d_{C} \in \Delta^{*}$

2) if $d \in \Delta^{*}, d^{\prime} \in \Delta^{\mathcal{I}}, 0 \leq i \leq n$, and $\exists R .\left(\Gamma_{C} \sqcap f_{i}: \Psi_{i}(D)\right) \in \operatorname{sub}(\Psi(C))$ such that

$$
\begin{aligned}
& -f_{i}^{\mathcal{I}}(d) \in\left(\exists R .\left(\Gamma_{C} \sqcap f_{i}: \Psi_{i}(D)\right)\right)^{\mathcal{I}}, \\
& \text { - }\left(f_{i}^{\mathcal{I}}(d), d^{\prime}\right) \in R^{\mathcal{I}}, \text { and } \\
& \text { - } d^{\prime} \in\left(\Gamma_{C} \sqcap f_{i}: \Psi_{i}(D)\right)^{\mathcal{I}},
\end{aligned}
$$

then $d^{\prime} \in \Delta^{*}$;

3) if $d \in \Delta^{*}, d_{1}, \ldots, d_{k} \in \Delta^{\mathcal{I}}, 0 \leq i \leq n, X \in \operatorname{sub}(\Psi(C))$, and $p^{*}$ is a path such that

$$
-d=d_{1},
$$

- $X$ is of the form $\Phi_{i}(p): f_{i}: \Psi_{i}(D) \sqcap \Gamma_{C}^{i}(p)$, and $p^{*}=p$,

$X$ is of the form $\Phi_{i}(p) \downarrow \Phi_{i}(q) \sqcap \Gamma_{C}^{i}(p) \sqcap \Gamma_{C}^{i}(q)$, and $p^{*} \in\{p, q\}$, or

$X$ is of the form $\Phi_{i}(p) \uparrow \Phi_{i}(q) \sqcap \Gamma_{C}^{i}(p) \sqcap \Gamma_{C}^{i}(q)$, and $p^{*} \in\{p, q\}$,

- $f_{i}^{\mathcal{I}}(d) \in X^{\mathcal{I}}$,

- $p^{*}=\gamma_{1} \circ \cdots \circ \gamma_{k-1}$, and

- $\left(f_{i}^{\mathcal{I}}\left(d_{\ell}\right), d_{\ell+1}\right) \in \gamma_{\ell}^{\mathcal{I}}$ for $1 \leq \ell<k$,

then $d_{1}, \ldots, d_{k} \in \Delta^{*}$.

Obviously, the sub-interpretation of $\mathcal{I}$ induced by $\Delta^{*}$ is rooted by $d_{C}$. Moreover, it is not hard to show that $\Delta^{*} \subseteq \Gamma_{C}^{\mathcal{I}}$ : 
$-d_{C} \in \Gamma_{C}^{\mathcal{I}}$ by definition of $\Psi(C)$.

- if $d^{\prime} \in \Delta^{*}$ due to Property 2 , then $d^{\prime} \in \Gamma_{C}$ by choice of $d^{\prime}$;

- Let $d_{1}, \ldots, d_{k} \in \Delta^{*}$ due to Property 3. Using the definition of the concept $\Gamma_{C}^{i}(p)$ and the fact that $f_{i}^{\mathcal{I}}\left(d_{1}\right) \in \Gamma_{C}^{i}\left(p^{*}\right)$, it is easily verified that $d_{j} \in \Gamma_{C}^{\mathcal{I}}$ for $1 \leq j \leq k$.

We now define a $\mathcal{T} \mathcal{L}-\mathcal{A L C F}$ interpretation $\mathcal{J}$. For convenience, we set

$$
t_{i}:=\operatorname{time}^{\mathcal{I}}\left(f_{i}^{\mathcal{I}}\left(d_{C}\right)\right), \text { for } 0 \leq i \leq n
$$

and use this abbreviation for the remainder of the proof. Now set

$$
\begin{aligned}
\Delta^{\mathcal{J}} & :=\Delta^{*} \\
A^{\mathcal{J}} & :=\left\{(t, d) \mid d \in \Delta^{\mathcal{J}}, t=t_{i}, \text { and } f_{i}^{\mathcal{I}}(d) \in A^{\mathcal{I}} \text { for some } i \leq n\right\} \\
R^{\mathcal{J}} & :=\left\{\left(t, d, d^{\prime}\right) \mid d, d^{\prime} \in \Delta^{\mathcal{J}}, t=t_{i}, \text { and }\left(f_{i}^{\mathcal{I}}(d), d^{\prime}\right) \in R^{\mathcal{I}} \text { for some } i \leq n\right\} \\
f^{\mathcal{J}} & :=\left\{\left(t, d, d^{\prime}\right) \mid d, d^{\prime} \in \Delta^{\mathcal{J}}, t=t_{i}, \text { and } f^{\mathcal{I}}\left(\left(f_{i}^{\mathcal{I}}(d)\right)=d^{\prime} \text { for some } i \leq n\right\}\right. \\
\star g^{\mathcal{J}} & :=\left\{\left(d, d^{\prime}\right) \mid d, d^{\prime} \in \Delta^{\mathcal{J}} \text { and } \star g^{\mathcal{I}}\left(f_{0}^{\mathcal{I}}(d)\right)=d^{\prime}\right\}
\end{aligned}
$$

We now prove some important properties of $\mathcal{J}$. Note that the first property implies that the interpretation of non-parametric features in $\mathcal{J}$ is functional as required (the interpretation of parametric features is obviously also functional, but does not depend on the following property).

1) For all $d \in \Delta^{*}$ and all $i, j$ with $t_{i}=t_{j}$, we have $f_{i}^{\mathcal{I}}(d)=f_{j}^{\mathcal{I}}(d)$.

Proof: By definition of $\Omega^{\prime}, t_{i}=t_{j}$ implies $d_{C} \in A_{i, j}$. Since $\Delta^{*}$ is rooted by $d_{C}$ and $\Delta^{*} \subseteq \Gamma_{C}^{\mathcal{I}}$, the definition of $\Gamma_{C}$ (second/third line) yields that $\Delta^{*} \subseteq A_{i, j}^{\mathcal{I}}$. Again by definition of $\Gamma_{C}$ (first line) and since $\Delta^{*} \subseteq \Gamma_{C}^{\mathcal{I}}$, this implies $f_{i}^{\mathcal{I}}(d)=f_{j}^{\mathcal{I}}(d)$ for all $d \in \Delta^{*}$.

2) For all $d \in \Delta^{*}$, all $i$ with $1 \leq i \leq n$, and all parametric features $\star g$, either $\star g^{\mathcal{I}}\left(f_{i}^{\mathcal{I}}(d)\right)$ and $\star g^{\mathcal{I}}\left(f_{0}^{\mathcal{I}}(d)\right)$ are both undefined, or $\star g^{\mathcal{I}}\left(f_{i}^{\mathcal{I}}(d)\right)=\star g^{\mathcal{I}}\left(f_{0}^{\mathcal{I}}(d)\right)$.

The proof is easy by considering the fact that $\Delta^{*} \subseteq \Gamma_{C}^{\mathcal{I}}$ together with the last line of the definition of $\Gamma_{C}$.

3) Let $d, d^{\prime} \in \Delta^{*}, p$ be a path not containing the features $f_{1}, \ldots, f_{n}$, and $0 \leq i \leq$ $n$. Then $d^{\prime} \in\left(\Phi_{i}(p)\right)^{\mathcal{I}}\left(f_{i}^{\mathcal{I}}(d)\right)$ iff $p_{t_{i}}^{\mathcal{J}}(d)=d^{\prime}$.

The proof is by induction on the length of $p$. For the induction start, let $p$ be of length one, i.e. $p=\gamma$. Then $\Phi_{i}(p)=\gamma$.

First, assume that $\gamma$ is a non-parametric feature. Then, $d^{\prime}=\gamma^{\mathcal{I}}\left(f_{i}^{\mathcal{I}}(d)\right)$ implies $\left(t_{i}, d, d^{\prime}\right) \in \gamma^{\mathcal{J}}$ by definition of $\mathcal{J}$, and thus the "only if" direction holds. For the "if" direction, assume that $\left(t_{i}, d, d^{\prime}\right) \in \gamma^{\mathcal{J}}$. Then there is a $j$ with $0 \leq j \leq n$ such that $t_{i}=t_{j}$ and $\gamma^{\mathcal{I}}\left(f_{j}^{\mathcal{I}}(d)\right)=d^{\prime}$. By Property 1 , we have $f_{j}^{\mathcal{I}}(d)=\bar{f}_{i}^{\mathcal{I}}(d)$ and thus $\gamma^{\mathcal{I}}\left(f_{i}^{\mathcal{I}}(d)\right)=d^{\prime}$ as required.

Let now $\gamma$ be a parametric feature. Then, $\gamma^{\mathcal{I}}\left(f_{i}^{\mathcal{I}}(d)\right)=d^{\prime}$ iff (by Property 2) $\gamma^{\mathcal{I}}\left(f_{0}^{\mathcal{I}}(d)\right)=d^{\prime}$ iff $\gamma^{\mathcal{J}}(d)=d^{\prime}$. 
Now for the induction step. Let $p=q \circ \gamma$. Then $\Phi_{i}(p)=\Phi_{i}(q) \circ f_{i} \circ \gamma$. Assume that $\left(\Phi_{i}(p)\right)^{\mathcal{I}}\left(f_{i}^{\mathcal{I}}(d)\right)=d^{\prime}$. Then there is a $d^{\prime \prime}$ with $\left(\Phi_{i}(q)\right)^{\mathcal{I}}\left(f_{i}^{\mathcal{I}}(d)\right)=d^{\prime \prime}$ and $\gamma^{\mathcal{I}}\left(f_{i}^{\mathcal{I}}\left(d^{\prime \prime}\right)\right)=d^{\prime}$. By IH, we obtain $q_{t_{i}}^{\mathcal{J}}(d)=d^{\prime \prime}$. To prove that $p_{t_{i}}^{\mathcal{J}}(d)=d^{\prime}$, it thus remains to show that $\gamma_{t_{i}}^{\mathcal{J}}\left(d^{\prime \prime}\right)=d^{\prime}$. In both the non-parametric and the parametric case, this can be done exactly as in the induction start (using the fact that $\gamma^{\mathcal{I}}\left(f_{i}^{\mathcal{I}}\left(d^{\prime \prime}\right)\right)=d^{\prime}$ ).

Vice-versa, assume that $p_{t_{i}}^{\mathcal{J}}(d)=d^{\prime}$. Then there is a $d^{\prime \prime}$ such that $q_{t_{i}}^{\mathcal{J}}(d)=d^{\prime \prime}$ and $\gamma_{t_{i}}^{\mathcal{J}}\left(d^{\prime \prime}\right)=d^{\prime}$. By IH, the former yields $\left(\Phi_{i}(q)\right)^{\mathcal{I}}\left(f_{i}^{\mathcal{I}}(d)\right)=d^{\prime \prime}$. It thus remains to show that $\gamma^{\mathcal{I}}\left(f_{i}^{\mathcal{I}}\left(d^{\prime \prime}\right)\right)=d^{\prime}$, which can again be done as in the induction start (using the fact that $\left.\gamma_{t_{i}}^{\mathcal{J}}\left(d^{\prime \prime}\right)=d^{\prime}\right)$.

We now prove the following, central claim:

Claim 5. - For all $d \in \Delta^{\mathcal{J}}, 0 \leq i \leq n$, and $D \in \operatorname{sub}(C)$, we have that $f_{i}^{\mathcal{I}}(d) \in$ $\Psi_{i}(D)^{\mathcal{I}}$ implies $d \in D_{t_{i}}^{\mathcal{J}}$.

This claim easily yields the desired result: since $C$ is in normal form, it is of the form

$$
C=\diamond(\bar{X}) \overline{T c} \cdot Q_{0} \sqcap Q_{1} @ X_{1} \sqcap \ldots \sqcap Q_{n} @ X_{n},
$$

with $\bar{X}=\left\{X_{1}, \ldots, X_{n}\right\}$. We define a variable assignment $\mathcal{W}$ for by setting $\mathcal{W}\left(X_{i}\right):=t_{i}$ for $1 \leq i \leq n$. Since $d_{C} \in \alpha(\overline{T c})^{\mathcal{I}}$, we have $\mathcal{W} \in\langle\bar{X}, \overline{T c}\rangle_{\sharp \mapsto t_{0}}^{\mathcal{E}}$. Using the claim, it is then readily verified that

$$
d_{C} \in\left(Q_{0} \sqcap Q_{1} @ X_{1} \sqcap \ldots \sqcap Q_{n} @ X_{n}\right)_{\mathcal{W}, t_{0}, \emptyset}^{\mathcal{J}} \cdot
$$

Thus, $d_{C} \in C_{t_{0}}^{\mathcal{J}}$ and $C$ is $\mathcal{T} \mathcal{L}-\mathcal{A L C \mathcal { F }}$ satisfiable as required. The proof of the claim is by structural induction:

- $D$ is a concept name. Then, $\Psi_{i}(D)=A$ and $\left(t_{i}, d\right) \in A^{\mathcal{J}}$ is an immediate consequence of the definition of $\mathcal{J}$.

$-D=\neg A$ ( $A$ is a concept name since $C$ is in NNF). Then, $\Psi_{i}(D)=\neg A$. Suppose $f_{i}^{\mathcal{I}}(d) \notin A^{\mathcal{I}}$ and $\left(t_{i}, d\right) \in A^{\mathcal{J}}$. Then there is a $j$ with $0 \leq j \leq n$ such that $t_{i}=t_{j}$ and $f_{j}^{\mathcal{I}}(d) \in A^{\mathcal{I}}$. By Property 1 , we have $f_{j}^{\mathcal{I}}(d)=f_{i}^{\mathcal{I}}(d)$. Thus, $f_{i}^{\mathcal{I}}(d) \in A^{\mathcal{I}}$, which is a contradiction.

$-D=D_{1} \sqcap D_{2}$. Easy using IH and the semantics.

- $D=D_{1} \sqcup D_{2}$. Easy using IH and the semantics.

- $D=\exists R . E$. Then, $\Psi_{i}(D)=\exists R$. $\left(\Gamma_{C} \sqcap f_{i}: \Psi_{i}(E)\right)$. Since $f_{i}^{\mathcal{I}}(d) \in \Psi_{i}(D)^{\mathcal{I}}$, there is a $d^{\prime}$ such that $\left(f_{i}^{\mathcal{I}}(d), d^{\prime}\right) \in R^{\mathcal{I}}$ and $d^{\prime} \in\left(\Gamma_{C} \sqcap f_{i}: \Psi_{i}(E)\right)^{\mathcal{I}}$. By definition of $\Delta^{*}$, we thus have $d^{\prime} \in \Delta^{*}$. Moreover, $f_{i}^{\mathcal{I}}\left(d^{\prime}\right) \in \Psi_{i}(E)^{\mathcal{I}}$. By definition of $R^{\mathcal{J}}$, we obtain $\left(t_{i}, d, d^{\prime}\right) \in R^{\mathcal{J}}$. By IH and since $f_{i}^{\mathcal{I}}\left(d^{\prime}\right) \in \Psi_{i}(E)^{\mathcal{I}}$, we get $d^{\prime} \in E_{t_{i}}^{\mathcal{J}}$. Thus, $d \in D_{t_{i}}^{\mathcal{J}}$.

$-D=\forall R . E$. Then, $\Psi_{i}(D)=\forall R .\left(f_{i}: \Psi_{i}(E)\right)$. Let $\left(t_{i}, d, d^{\prime}\right) \in R^{\mathcal{J}}$. Then there is a $j$ with $0 \leq j \leq n$ such that $t_{i}=t_{j}$ and $\left(f_{j}^{\mathcal{I}}(d), d^{\prime}\right) \in R^{\mathcal{I}}$. By Property 1 , we have $f_{j}^{\mathcal{I}}(d)=f_{i}^{\mathcal{I}}(d)$ and hence $\left(f_{i}^{\mathcal{I}}(d), d^{\prime}\right) \in R^{\mathcal{I}}$. Since $f_{i}^{\mathcal{I}}(d) \in \Psi_{i}(D)^{\mathcal{I}}$, we thus have $d^{\prime} \in\left(f_{i}: \Psi_{i}(E)\right)^{\mathcal{I}}$ and $f_{i}^{\mathcal{I}}\left(d^{\prime}\right) \in \Psi_{i}(E)^{\mathcal{I}}$. Thus, IH yields $d^{\prime} \in E_{t_{i}}^{\mathcal{J}}$ as required. 
$-D=p: E$. Then, $\Psi_{i}(D)=\Phi_{i}(p): f_{i}: \Psi_{i}(E) \sqcap \Gamma_{C}^{i}(p)$. Since $f_{i}^{\mathcal{I}}(d) \in$ $\Psi_{i}(D)$, there is a $d^{\prime} \in \Delta^{\mathcal{I}}$ such that $\left(\Phi_{i}(p)\right)^{\mathcal{I}}\left(f_{i}^{\mathcal{I}}(d)\right)=d$ and $f_{i}^{\mathcal{I}}(d) \in \Psi_{i}(E)^{\mathcal{I}}$. By Property 3 , we thus have $p_{t_{i}}^{\mathcal{J}}(d)=d^{\prime}$, and IH yields $d^{\prime} \in E_{t_{i}}^{\mathcal{J}}$. Summing up, we obtain $d \in D_{t_{i}}^{\mathcal{J}}$.

$-D=p \downarrow q$. Then, $\Psi_{i}(D)=\Phi_{i}(p) \downarrow \Phi_{i}(q) \sqcap \Gamma_{C}^{i}(p) \sqcap \Gamma_{C}^{i}(q)$. Since $f_{i}^{\mathcal{I}}(d) \in$ $\Psi_{i}(D)^{\mathcal{I}}$, there is a $d^{\prime} \in \Delta^{\mathcal{I}}$ such that

$$
\left(\Phi_{i}(p)\right)^{\mathcal{I}}\left(f_{i}^{\mathcal{I}}(d)\right)=\left(\Phi_{i}(q)\right)^{\mathcal{I}}\left(f_{i}^{\mathcal{I}}(d)\right)=d .
$$

By definition of $\Delta^{*}$, we have $d^{\prime} \in \Delta^{*}$. By Property 3, we have $p_{t_{i}}^{\mathcal{J}}(d)=q_{t_{i}}^{\mathcal{J}}(d)=d^{\prime}$, and thus $d \in D_{t_{i}}^{\mathcal{J}}$ as required.

$-D=p \uparrow q$. Then, $\Psi_{i}(D)=\Phi_{i}(p) \uparrow \Phi_{i}(q) \sqcap \Gamma_{C}^{i}(p) \sqcap \Gamma_{C}^{i}(q)$. Analogous to the previous case.

$-D=p \uparrow$. Then, $\Psi_{i}(D)=\Phi_{i}(p) \uparrow$. Let $f_{i}^{\mathcal{I}}(d) \in \Psi_{i}(D)^{\mathcal{I}}$, and assume that $d \notin D_{t_{i}}^{\mathcal{J}}$. Then there is a $d^{\prime} \in \Delta^{\mathcal{J}}$ such that $p_{t_{i}}^{\mathcal{J}}(d)=d^{\prime}$. By Property 3 , we thus have $\left(\Phi_{i}(p)\right)^{\mathcal{I}}\left(f_{i}^{\mathcal{I}}(d)\right)=d^{\prime}$, which is a contradiction.

Proposition 6. - Let $C$ be a $\mathcal{T} \mathcal{L}$ - $\mathcal{A L C \mathcal { F }}$ concept in normal form. Then $\mathcal{T} \mathcal{L}$ - $\mathcal{A} \mathcal{L C} \mathcal{F}$ satisfi ability of $C$ implies $\mathcal{A L C \mathcal { F }}(A)$ satisfi ability of $\Psi(C)$.

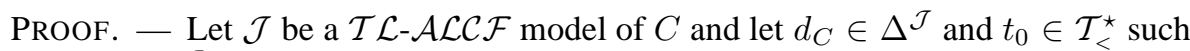
that $d_{C} \in C_{t_{0}}^{\mathcal{J}}$. Let

$$
C=\diamond(\bar{X}) \overline{T c} \cdot Q_{0} \sqcap Q_{1} @ X_{1} \sqcap \ldots \sqcap Q_{n} @ X_{n}
$$

with $\bar{X}=\left\{X_{1}, \ldots, X_{n}\right\}$. By the semantics, there exists a variable assignment $\mathcal{W} \in$ $\langle\bar{X}, \overline{T c}\rangle_{\sharp \rightarrow t_{0}}^{\mathcal{E}}$ such that

$$
d_{C} \in\left(Q_{0} \sqcap Q_{1} @ X_{1} \sqcap \ldots \sqcap Q_{n} @ X_{n}\right)_{\mathcal{W}, t_{0}, \emptyset}^{\mathcal{J}} .
$$

In the remainder of this proof, we use $X_{0}=\sharp$, and $t_{i}$ to denote $\mathcal{W}\left(X_{i}\right)$, for $1 \leq i \leq n$. We now construct an $\mathcal{A} \mathcal{L C} \mathcal{F}$-interpretation $\mathcal{I}$ :

$$
\begin{aligned}
\Delta^{\mathcal{I}} & :=\Delta^{\mathcal{J}} \cup\left\{\left(d, t_{i}\right) \mid d \in \Delta^{\mathcal{J}} \text { and } 0 \leq i \leq n\right\} \\
A^{\mathcal{I}} & :=\left\{(d, t) \mid(t, d) \in A^{\mathcal{J}} \text { and } t=t_{i} \text { for some } i \leq n\right\} \\
f^{\mathcal{I}} & :=\left\{\left((d, t), d^{\prime}\right) \mid\left(t, d, d^{\prime}\right) \in f^{\mathcal{J}} \text { and } t=t_{i} \text { for some } i \leq n\right\} \\
\star g^{\mathcal{I}} & :=\left\{\left(\left(d, t_{i}\right), d^{\prime}\right) \mid\left(d, d^{\prime}\right) \in \star g^{\mathcal{J}} \text { and } 0 \leq i \leq n\right\} \\
R^{\mathcal{I}} & :=\left\{\left((d, t), d^{\prime}\right) \mid\left(t, d, d^{\prime}\right) \in R^{\mathcal{J}} \text { and } t=t_{i} \text { for some } i \leq n\right\} \\
t i m e^{\mathcal{I}} & :=\left\{\left(\left(d_{C}, t_{i}\right), t_{i}\right) \mid 0 \leq i \leq n\right\} \\
A_{j, \ell}^{\mathcal{I}} & := \begin{cases}\Delta^{\mathcal{J}} & \text { if } t_{j}=t_{\ell} \\
\emptyset & \text { otherwise }\end{cases} \\
f_{i}^{\mathcal{I}} & :=\left\{\left(d,\left(d, t_{i}\right)\right) \mid d \in \Delta^{\mathcal{J}} \text { and } 0 \leq i \leq n\right\}
\end{aligned}
$$


for all concept names $A, A_{i, j}$, non-parametric features $f$, reserved features $f_{i}$, parametric features $\star g$, role names $R$, and $j, \ell \in\{0, \ldots, n\}$. We show that $d_{C} \in \Psi(C)^{\mathcal{I}}$. To this end, it is readily verified that $d_{C} \in\left(\alpha(\overline{\overline{T c}}) \sqcap \Gamma_{C} \sqcap \Omega^{\prime}\right)^{\mathcal{I}}$. It thus remains to show that $d_{C} \in \Omega^{\mathcal{I}}$. This is obviously an immediate consequence of $(*), \mathcal{I}$ 's interpretation of the $f_{i}$ features, and the following claim:

Claim 7. - For all $d \in \Delta^{\mathcal{J}}, 0 \leq i \leq n$, and $D \in \operatorname{sub}(C)$, we have that $d \in D_{t_{i}}^{\mathcal{J}}$ implies $\left(d, t_{i}\right) \in \Psi_{i}(D)^{\mathcal{I}}$.

Before we prove the claim, let us state three useful properties of $\Delta^{\mathcal{I}}$ :

1) $\Delta^{\mathcal{J}} \subseteq \Gamma_{C}^{\mathcal{I}}$, as it is easily verified by considering the definitions of both $A_{j, \ell}^{\mathcal{I}}$ and $\star g^{\mathcal{I}}$.

2) For all $d \in \Delta^{\mathcal{J}}$, paths $p$ not containing the features $f_{0}, \ldots, f_{n}$, and $i \in$ $\{0, \ldots, n\}$, the following holds: if there is a $d^{\prime}$ with $p_{t_{i}}^{\mathcal{J}}(d)=d^{\prime}$, then $\left(d, t_{i}\right) \in$ $\Gamma_{C}^{i}(p)^{\mathcal{I}}$.

The proof is by induction on the length of $p$, using Property 1 . Details are left to the reader.

3) Let $d, d^{\prime} \in \Delta^{\mathcal{J}}, p$ be a path not containing the features $f_{0}, \ldots, f_{n}$, and $i \in$ $\{0, \ldots, n\}$. Then $p_{t_{i}}^{\mathcal{J}}(d)=d^{\prime}$ iff $\left(\Phi_{i}(p)\right)^{\mathcal{I}}\left(\left(d, t_{i}\right)\right)=d^{\prime}$.

The proof is again by induction on the length of $p$. Details are left to the reader.

We now proof the claim by structural induction:

- $D$ is a concept name. Then, $\Psi_{i}(D)=A$, and $\left(d, t_{i}\right) \in D^{\mathcal{I}}$ is an immediate consequence of the definition of $\mathcal{J}$.

- $D=\neg A$ ( $A$ is a concept name since $C$ is in NNF). Then, $\Psi_{i}(D)=\neg A$. It is an immediate consequence of the definition of $A^{\mathcal{I}}$ that $\left(t_{i}, d\right) \notin A^{\mathcal{J}}$, which implies $\left(d, t_{i}\right) \notin A^{\mathcal{I}}$.

- $D=D_{1} \sqcap D_{2}$. Easy using IH and the semantics.

$-D=D_{1} \sqcup D_{2}$. Easy using IH and the semantics.

- $D=\exists R . E$. Then, $\Psi_{i}(D)=\exists R .\left(\Gamma_{C} \sqcap f_{i}: \Psi_{i}(E)\right)$. Since $d \in D_{t_{i}}^{\mathcal{J}}$, there is a $d^{\prime} \in \Delta^{\mathcal{J}}$ such that $\left(t_{i}, d, d^{\prime}\right) \in R^{\mathcal{J}}$ and $d^{\prime} \in E_{t_{i}}^{\mathcal{J}}$. By definition of $R^{\mathcal{I}}$, we obtain $\left(\left(d, t_{i}\right), d^{\prime}\right) \in R^{\mathcal{I}}$. By IH, we get $\left(d^{\prime}, t_{i}\right) \in E^{\mathcal{I}}$. By the interpretation of the $f_{i}$ features, this yields $d^{\prime} \in\left(f_{i}: \Psi_{i}(E)\right)^{\mathcal{I}}$. By Property 1 , we get $\left(d, t_{i}\right) \in \Psi_{i}(D)^{\mathcal{I}}$.

$-D=\forall R . E$. Then, $\Psi_{i}(D)=\forall R .\left(f_{i}: \Psi_{i}(E)\right)$. Let $\left(\left(d, t_{i}\right), d^{\prime}\right) \in R^{\mathcal{I}}$. By definition of $R^{\mathcal{I}}$, we have $d^{\prime} \in \Delta^{\mathcal{J}}$ and $\left(t_{i}, d, d^{\prime}\right) \in R^{\mathcal{J}}$. Since $d \in D_{t_{i}}^{\mathcal{J}}$, we thus have $d^{\prime} \in E_{t_{i}}^{\mathcal{J}}$. Thus, IH yields $\left(d^{\prime}, t_{i}\right) \in \Psi_{i}(E)^{\mathcal{I}}$. By the interpretation of the $f_{i}$ features, this yields $d^{\prime} \in\left(f_{i}: \Psi_{i}(E)\right)^{\mathcal{I}}$ as required.

- $D=p: E$. Then, $\Psi_{i}(D)=\Phi_{i}(p): f_{i}: \Psi_{i}(E) \sqcap \Gamma_{C}^{i}(p)$. Since $d \in D_{t_{i}}^{\mathcal{J}}$, there are is a $d^{\prime} \in \Delta^{\mathcal{J}}$ such that $p_{t_{i}}^{\mathcal{J}}(d)=d^{\prime}$ and $d^{\prime} \in E_{t_{i}}^{\mathcal{J}}$. By Property 3, we have $\Phi_{i}(p)^{\mathcal{I}}\left(\left(d, t_{i}\right)\right)=d^{\prime}$ and $\mathrm{IH}$ yields $\left(d^{\prime}, t_{i}\right) \in \Psi_{i}(E)^{\mathcal{I}}$. Thus, by the interpretation of the $f_{i}$ features we have $\left(d, t_{i}\right) \in\left(\Phi_{i}(p): f_{i}: \Psi_{i}(E)\right)^{\mathcal{I}}$. To verify that $\left(d, t_{i}\right) \in$ $\Psi_{i}(D)^{\mathcal{I}}$, it thus remains to show that $\left(d, t_{i}\right) \in \Gamma_{C}^{i}(p)^{\mathcal{I}}$, which is true because of 


\section{Property 2.}

- $D=p \downarrow q$. Then, $\Psi_{i}(D)=\Phi_{i}(p) \downarrow \Phi_{i}(q) \sqcap \Gamma_{C}^{i}(p) \sqcap \Gamma_{C}^{i}(q)$. Since $d \in D_{t_{i}}^{\mathcal{J}}$, there are is a $d^{\prime} \in \Delta^{\mathcal{J}}$ such that $p_{t_{i}}^{\mathcal{J}}(d)=q_{t_{i}}^{\mathcal{J}}(d)=d^{\prime}$. By Property 3, we have $\Phi_{i}(p)^{\mathcal{I}}\left(\left(d, t_{i}\right)\right)=\Phi_{i}(q)^{\mathcal{I}}\left(\left(d, t_{i}\right)\right)=d^{\prime}$. Thus, $\left(d, t_{i}\right) \in\left(\Phi_{i}(p) \downarrow \Phi_{i}(q)\right)^{\mathcal{I}}$. To verify that $\left(d, t_{i}\right) \in \Psi_{i}(D)^{\mathcal{I}}$, it thus remains to show that $\left(d, t_{i}\right) \in\left(\Gamma_{C}^{i}(p) \sqcap \Gamma_{C}^{i}(q)\right)^{\mathcal{I}}$, which is an easy consequence of Property 2.

- $D=p \uparrow q$. Then, $\Psi_{i}(D)=\Phi_{i}(p) \uparrow \Phi_{i}(q) \sqcap \Gamma_{C}^{i}(p) \sqcap \Gamma_{C}^{i}(q)$. Analogous to the previous case.

- $D=p \uparrow$. Then, $\Psi_{i}(D)=\Phi_{i}(p) \uparrow$. Let $d \in(p \uparrow)^{\mathcal{J}}$ and assume that $\left(d, t_{i}\right) \notin$ $\Psi_{i}(D)^{\mathcal{I}}$. Then there is a $d^{\prime} \in \Delta^{\mathcal{I}}$ such that $\Phi_{i}(p)^{\mathcal{I}}\left(\left(d, t_{i}\right)\right)=d^{\prime}$. By definition of $\Phi_{i}(p)$ and of $\mathcal{I}$, we have $d^{\prime} \in \Delta^{\mathcal{J}}$. By Property 3 , we obtain $p_{t_{i}}^{\mathcal{J}}(d)=d^{\prime}$, which is a contradiction.

Since satisfiability of $\mathcal{A L C F}(A)$ concepts is PSPACE-complete [LUT 02c], satisfiability of $\mathcal{A L C}$-concepts is PSPACE-hard, and $\mathcal{A L C}$ is a fragment of $\mathcal{T} \mathcal{L}-\mathcal{A L C F}$, we obtain the following theorem.

THEOREM 8. - Satisfi ability of $\mathcal{T} \mathcal{L}-\mathcal{A} \mathcal{L C} \mathcal{F}$ concepts is PSPACE-complete.

\section{Conclusions}

We have discussed the relationship between the two interval-based temporal DLs $\mathcal{T} \mathcal{L}-\mathcal{A L C F}$ and $\mathcal{A L C} \mathcal{F}(A)$, and found that the gap between the two different knowledge representation paradigms suggested by these logics can be bridged by a suitable translation. Based on this translation, we have presented a reduction from $\mathcal{T} \mathcal{L}-\mathcal{A} \mathcal{L C} \mathcal{F}$ concept satisfiability to $\mathcal{A L C F}(A)$ concept satisfiability that allowed us to determine the complexity of $\mathcal{T} \mathcal{L}-\mathcal{A L C} \mathcal{F}$ concept satisfiability as a PSPACE-complete problem. Moreover, the reduction allows to use the $\mathcal{A L C F}(A)$ tableau algorithm described in [LUT 02c] to be used for reasoning on $\mathcal{T} \mathcal{L}-\mathcal{A L C} \mathcal{F}$ concept expressions.

Concerning future work, the described reduction can be extended in at least two interesting directions:

(1) In this paper, we concentrated on the satisfiability of concepts. In description logics, an equally important reasoning task is the subsumption of concepts: a concept $C$ is subsumed by a concept $D$ if $C^{\mathcal{I}} \subset D^{\mathcal{I}}$ for all interpretations $\mathcal{I}$. In description logics with all Boolean operators, subsumption can be reduced to (un)satisfiability: $C$ is subsumed by $D$ iff $C \sqcap \neg D$ is unsatisfiable. Clearly, we cannot do this in $\mathcal{T} \mathcal{L}-\mathcal{A} \mathcal{L C} \mathcal{F}$ since full negation is not available in the temporal part. ${ }^{3}$ Moreover, our reduction cannot be used to decide $\mathcal{T} \mathcal{L}-\mathcal{A L C F}$ subsumption. Consider, for example, the concepts

3. Indeed, adding full negation to $\mathcal{T} \mathcal{L}-\mathcal{A L C} \mathcal{F}$ would result in undecidability. Still, it was shown in [ART 98] that subsumption of $\mathcal{T} \mathcal{L}$ - $\mathcal{A} \mathcal{L C} \mathcal{F}$-concepts is decidable. 


$$
\begin{aligned}
& C=\diamond(x)(\sharp \text { before } x) \cdot A @ x \\
& D=\diamond(x, y)(\sharp \text { before } x)(y \text { equals } x) . A @ y
\end{aligned}
$$

Then $C$ is subsumed by $D$ (actually, they are equivalent concepts), but $\Psi(C)$ is not subsumed by $\Psi(D)$ since $\Psi(C)$ has only two "reserved features" $f_{0}$ and $f_{1}$, while $\Psi(D)$ has three: $f_{0}, f_{1}$, and $f_{2}$. It would thus be interesting to extend the correspondence between $\mathcal{T} \mathcal{L}$ - $\mathcal{A} \mathcal{L C} \mathcal{F}$ and $\mathcal{A} \mathcal{L C F}(A)$ developed in this paper to concept subsumption.

(2) For the reduction, we consider the satisfiability of concepts without reference to so-called TBoxes. As modern DLs are usually equipped with TBoxes [BAA 03b], it would be worthwhile to add them to both $\mathcal{T} \mathcal{L}$ - $\mathcal{A} \mathcal{L C} \mathcal{F}$ and $\mathcal{A L C F}(A)$, and to extend our reduction accordingly. However, we cannot expect to obtain PSPACE-results:

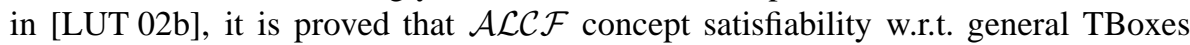
(also known as GCIs) is undecidable. Thus, the same holds for both $\mathcal{T} \mathcal{L}-\mathcal{A L C F}$ and $\mathcal{A L C} \mathcal{F}(A)$. Undecidability may be overcome by resorting to so-called acyclic TBoxes [BAA 03b]. However, as proved in [LUT 99], $\mathcal{A} \mathcal{L C} \mathcal{F}$ concept satisfiability w.r.t. acyclic TBoxes is NEXPTIME-hard. Clearly, this lower bound is inherited by $\mathcal{T} \mathcal{L}-\mathcal{A L C F}$ and $\mathcal{A L C} \mathcal{F}(A)$. A matching upper bound for $\mathcal{A L C F}(A)$ has been proved in [LUT 02c]. A similar bound for $\mathcal{T} \mathcal{L}-\mathcal{A L C \mathcal { F }}$ is yet to be established.

\section{References}

[ALL 83] ALLEN J., "Maintaining Knowledge about Temporal Intervals", Communications of the ACM, vol. 26, num. 11, 1983.

[All 91] Allen J. F., Kautz H. A., Pelavin R. N., Tenenberg J. D., Eds., Reasoning about Plans, Morgan Kaufmann, 1991.

[All 94] Allen J. F., Ferguson G., "Actions and Events in Interval Temporal Logic", Journal of Logic and Computation, vol. 4, num. 5, 1994, Special Issue on Actions and Processes.

[ART 94] ARtale A., Franconi E., "A Computational Account for a Description Logic of Time and Action", J.Doyle, E.S Andewall, P.Torasso, Eds., Proc. of the 4th International Conference on Principles of Knowledge Representation and Reasoning - KR94, Bonn, Germany, May 1994, Morgan Kaufmann, p. 3-14.

[ART 98] ARtale A., Franconi E., "A Temporal Description Logic for Reasoning about Actions and Plans", Journal of Artificial Intelligence Research (JAIR), vol. 9, 1998, p. 463506.

[ART 99a] ARtale A., Franconi E., 'Representing a robotic domain using temporal description logics", Artificial Intelligence in Engineering, Design, Analysis and Manifacturing, vol. 13, num. 9, 1999, p. 105-117, Cambridge University Press.

[ART 99b] ARtale A., Franconi E., "Temporal ER Modeling with Description Logics", Proceedings of the International Conference on Conceptual Modeling (ER'99), vol. 1728 of Lecture Notes in Computer Science, Springer-Verlag, 1999. 
[ART 01] ARtale A., Franconi E., "A Survey of Temporal Extensions of Description Logics", Annals on Mathematics and Artificial Intelligence (AMAI), vol. 30, num. 1-4, 2001, p. 171-210, Kluwer Academic Publishers.

[ART 02] Artale A., Franconi E., Wolter F., Zakharyaschev M., "A Temporal Description Logic for Reasoning about Conceptual Schemas and Queries", FLESCA S., Greco S., Leone N., IAnNi G., Eds., Proceedings of the 8th Joint European Conference on Logics in Artificial Intelligence (JELIA-O2), vol. 2424 of LNAI, Springer, 2002, p. $98-110$.

[ART 03] Artale A., Franconi E., Mandreoli F., 'Description Logics for Modeling of Dynamic Information", CHOMICKI J., VAN DER MEYDEn R., SAAKE G., Eds., Logics for Emerging Applications of Databases, Page 368, Springer-Verlag, 2003.

[BAA 00] BAAder F., SATtler U., 'Tableau Algorithms for Description Logics”, DyCKHOFF R., Ed., Proceedings of the International Conference on Automated Reasoning with Tableaux and Related Methods (Tableaux 2000), vol. 1847 of Lecture Notes in Artificial Intelligence, Springer-Verlag, 2000, p. 1-18.

[BAA 03a] BaAder F., KÜsters R., Wolter F., 'Extensions to Description Logics", BaAder F., McGuiness D. L., NARdi D., PATEl-Schneider P., Eds., The Description Logic Handbook: Theory, implementation and applications, Cambridge University Press, 2003.

[BAA 03b] BAader F., McGuiness D. L., NArdi D., PATEl-Schneider P., Eds., The Description Logic Handbook: Theory, implementation and applications, Cambridge University Press, 2003.

[BEN 95] Van Benthem J., "Temporal Logic", Gabbay D., Hogger C., Robinson J., Eds., Handbook of Logic in Artificial Intelligence and Logic Programming, Volume 4, p. 241-350, Oxford Science Publishers, 1995.

[BET 97] BETTINI C., "Time-dependent concepts: representation and reasoning using temporal description logics", Data \& Knowledge Engineering, vol. 22, 1997, p. 1-38.

[CHO 98] ChOmicki J., SAAKe G., Eds., Logics for Databases and Information Systems, Kluwer Academic Publisher, 1998.

[CHO 03] Chomicki J., VAn Der Meyden R., SAAKe G., Eds., Logic for Emerging Application of Databases, Springer-Verlag, 2003.

[EME 90] Emerson A., "Temporal and Modal Logic", VAn LeEuwen J., Ed., Handbook of Theoretical Computer Science, vol. B, p. 995-1072, Elsevier, 1990.

[GAB 94] GabBay D. M., Hodkinson I. M., Reynolds M. A., Eds., Temporal Logic: Mathematical Foundations and Computational Aspects, Volume 1, Oxford University Press, Logic Guides 28, 1994.

[GAB 03] Gabbay D. M., Kurucz A., Wolter F., Zakharyaschev M., ManyDimensional Modal Logics: Theory and Applications, Num. 148 Studies in Logic and the Foundations of Mathematics, Elsevier, 2003.

[GAB ar] GabBay D., Fisher M., VILA L., Eds., Handbook of Time and Temporal Reasoning in Artificial Intelligence, Elsevier, To appear.

[GOR 03a] Goranko V., Montanari A., Sciavicco G., 'Propositional Interval Neighborhood Temporal Logics”, Journal of Universal Computer Science, vol. 9, num. 9, 2003.

[GOR 03b] Goranko V., Montanari A., Sciavicco G., "A Road Map on Interval Temporal Logics and Duration Calculi”, Goranko V., Montanari A., Eds., Proc. of the 
ESSLLI Workshop on Interval Temporal Logics and Duration Calculi, 2003, p. 1-40.

[HAA 01] HAARSLEV V., MÖller R., 'RACER system description”, GorÉ R., LEITSCH A., NipKow T., Eds., Proceedings of the First International Joint Conference on Automated Reasoning (IJCAR'01), num. 2083 Lecture Notes in Artifical Intelligence, SpringerVerlag, 2001, p. 701-705.

[HAL 91] Halpern J. Y., Shoham Y., "A Propositional Modal Logic of Time Intervals", Journal of ACM, vol. 38, num. 4, 1991, p. 935-962.

[HOL 90] Hollunder B., NutT W., 'Subsumption Algorithms for Concept Languages", DFKI Research Report num. RR-90-04, 1990, German Research Center for Artificial Intelligence (DFKI), Kaiserslautern, Germany.

[HOR 00] Horrocks I., Sattler U., Tobies S., 'Practical Reasoning for Very Expressive Description Logics", Logic Journal of the IGPL, vol. 8, num. 3, 2000, p. 239-264.

[LUT 97] Lutz C., HAARslev V., Möller R., "A Concept Language with Role-Forming Predicate Restrictions", Technical Report num. FBI-HH-M-276/97, 1997, University of Hamburg, Computer Science Department, Hamburg.

[LUT 99] Lutz C., 'Complexity of Terminological Reasoning Revisited”, Ganzinger H., MCAllester D., Voronkov A., Eds., Proceedings of the 6th International Conference on Logic for Programming and Automated Reasoning (LPAR'99), num. 1705 Lecture Notes in Artificial Intelligence, Springer-Verlag, 1999, p. 181-200.

[LUT 01] Lutz C., Sturm H., Wolter F., Zakharyaschev M., "Tableaux for Temporal Description Logic with Constant Domain”, Goré R., LeITSCH A., NiPKOW T., Eds., Proceedings of the First International Joint Conference on Automated Reasoning (IJCAR'01), num. 2083 Lecture Notes in Artifical Intelligence, Springer-Verlag, 2001, p. 121-136.

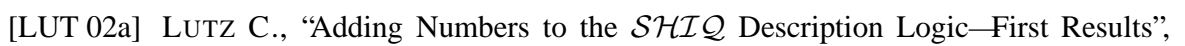
Proc. of the 8th International Conference on Principles of Knowledge Representation and Reasoning - KR2002, Morgan Kaufman, 2002, p. 191-202.

[LUT 02b] LUtz C., "The Complexity of Reasoning with Concrete Domains", PhD thesis, LuFG Theoretical Computer Science, RWTH Aachen, Germany, 2002.

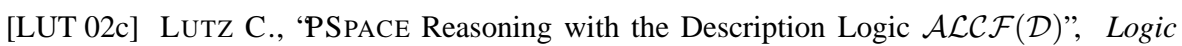
Journal of the IGPL, vol. 10, num. 5, 2002, p. 535-568.

[LUT 03] LUTZ C., 'Description Logics with Concrete Domains-A Survey", BALBIANI P., Suzuki N.-Y., Wolter F., Zakharyaschev M., Eds., Advances in Modal Logics Volume 4, King's College Publications, 2003, p. 265-296.

[LUT ar] LUTz C., 'Combining Interval-based Temporal Reasoning with General TBoxes", Artificial Intelligence, To appear.

[NEB 95] Nebel B., BÜRCKERT H.-J., 'Reasoning about temporal relations: a maximal tractable subclass of Allen's interval algebra", Journal of the ACM, vol. 42, 1995, p. 4366.

[PAT 99] Patel-Schneider P., 'DlP', LAmbrix P., Borgida A., LenZerini M., Möller R., PATEl-SchneIder P., Eds., Proceedings of the International Workshop on Description Logics (DL'99), num. 22 CEUR-WS (http://ceur-ws.org/Vol-22/), 1999, p. 913.

[PNU 86] PNUELI A., "Application of Temporal Logic to the Specification and Verification of Reactive Systems: A Survey of Current Trends", Current Trends in Concurrency, vol. 224 of Lecture Notes in Computer Science, p. 510-584, Springer-Verlag, 1986. 
[SCH 90] SCHMiEdel A., "Temporal Terminological Logic", DiETTERICH, TOM; SwARTOUT W., Ed., Proceedings of the 8th National Conference on Artificial Intelligence (AAAI'90), MIT Press, 1990, p. 640-645.

[SCH 93] SChILD K. D., "Combining Terminological Logics with Tense Logic", Filgueiras M., Damas L., Eds., Progress in Artificial Intelligence - 6th Portuguese Conference on Artificial Intelligence, EPIA'93, vol. 727 of Lecture Notes in Artificial Intelligence, Springer-Verlag, 1993, p. 105-120.

[STU 01] StUrm H., Wolter F., "A tableau calculus for temporal description logic: The expanding domain case", Journal of Logic and Computation, vol. 12, num. 5, 2001.

[VEN 90] VENEMA Y., 'Expressiveness and Completeness of an Interval Tense Logic”, Notre Dame Journal of Formal Logic, vol. 31, num. 4, 1990, p. 529-547.

[VIL 90] Vilain M., Kautz H., Van Beek P., 'Constraint Propagation Algorithms for Temporal Reasoning: A Revised Report”, WELD D. S., DE KLEER J., Eds., Readings in Qualitative Reasoning about Physical Systems, p. 373-381, Morgan Kaufmann, 1990.

[WOL 98] Wolter F., ZaKharyaschev M., 'Satisfiability Problem in Description Logics with Modal Operators", Cohn A. G., Schubert L., Shapiro S. C., Eds., Proc. of the 6th International Conference on Principles of Knowledge Representation and Reasoning KR98, p. 512-523, Morgan Kaufmann, San Francisco, CA, USA, 1998.

[WOL 99] WOlter F., ZaKharyaschev M., "Temporalizing description logic", GabBay D., DE RiJKe M., Eds., Frontiers of Combining Systems, p. 379 - 402, Studies Press/Wiley, 1999. 